\title{
GLOBAL EXISTENCE AND ASYMPTOTIC BEHAVIOR TO THE SOLUTIONS OF 1-D LYUMKIS ENERGY TRANSPORT MODEL FOR SEMICONDUCTORS
}

\author{
BY \\ LI CHEN (Department of Mathematical Sciences, Tsinghua University, Beijing 100084, P. R. \\ China), \\ LING HSIAO (Academy of Mathematics and System Sciences, CAS, Beijing 100080, P. R. \\ China), \\ AND
}

YONG LI (Academy of Mathematics and System Sciences, CAS, Beijing 100080, P. R. China)

\begin{abstract}
The global existence and asymptotic behavior of smooth solutions to the initial-boundary value problem for the 1-D Lyumkis energy transport model in semiconductor science is studied. When the boundary is insulated, the smooth solution of the problem converges to a stationary solution of the drift diffusion equations, exponentially fast as $t \rightarrow \infty$.
\end{abstract}

1. Introduction. The energy transport models in semiconductor science can be derived directly from the Boltzmann equation in the diffusion limit $[1,2,8,13$, 12], or obtained formally from the hydrodynamic equations by neglecting certain terms [13]. The common form of the energy transport model is governed by the system

$$
\begin{aligned}
& \frac{\partial n}{\partial t}+\operatorname{div} J_{1}=0 \\
& \frac{\partial E}{\partial t}+\operatorname{div} J_{2}=\nabla V \cdot J_{1}+W(\mu, T) \text { in } \Omega \\
& \lambda^{2} \triangle V=n-C(x)
\end{aligned}
$$

Received September 11, 2002.

2000 Mathematics Subject Classification. Primary 35M10, 35K55, 35K65, 78A35.

Key words and phrases. Lyumkis energy transport model, strong parabolic condition, global existence, asymptotic behavior.

Supported by the Special Funds of State Major Basic Research Projects (Grant No. 1999075107) and the Innovation Funds of AMSS, CAS of China.

E-mail address: Ichen@math.tsinghua.edu.cn

E-mail address: hsiaol@mail.math.ac.cn

E-mail address: yli@mail.amss.ac.cn 
with

$$
\begin{aligned}
& J_{1}=-L_{11}\left(\nabla\left(\frac{\mu}{T}\right)-\frac{\nabla V}{T}\right)-L_{12} \nabla\left(-\frac{1}{T}\right), \\
& J_{2}=-L_{21}\left(\nabla\left(\frac{\mu}{T}\right)-\frac{\nabla V}{T}\right)-L_{22} \nabla\left(-\frac{1}{T}\right),
\end{aligned}
$$

where the unknowns $\mu, T$ are the chemical potential of the electrons and the electron temperature, respectively, $V$ is the electrostatic potential, $n$ is the electron density, $E$ is the density of the internal energy, $W(\mu, T)$ is the energy relaxation term, satisfying $W(\mu, T)\left(T-T_{0}\right) \leq 0$, where the positive constant $T_{0}$ is the lattice temperature, $J_{1}$ is the carrier flux density, $J_{2}$ is the energy flux density, or the heat flux, $L$ are the diffusion matrices, $\lambda$ is the scaled Debye length, and $C(x)$ is the doping profile which represents the background of the device. The expressions for $n, E, L$, and $W$ are constitutive relations. Various forms, corresponding to different models, are found in the literature.

In a parabolic band structure, the relations for $n$ and $E$ derived from the Boltzmann equation are

$$
n=T^{\frac{3}{2}} \exp \left\{\frac{\mu}{T}\right\}, \quad E=\frac{3}{2} n T .
$$

Several authors have recently studied stationary energy transport models $[6,9,14,4]$, and have obtained useful results. For the transient case, the first results on the existence of a weak solution and its large time behavior for a more general parabolic system were obtained by P. Degond, S. Génieys, and A. Jüngel [7]. They employed semidiscretization of time and used the entropy function under physically motivated Dirichlet-Neumann boundary conditions and initial conditions. Furthermore, these authors have established in [11] the regularity and uniqueness when the coefficient matrix $L$ depends solely on $x$. Unfortunately, however, in both [7] and [11] it is required that $L$ be uniformly positive definite, while the more interesting situation in physics arises when the coefficient matrix is merely positive definite. L. Chen and L. Hsiao [5] have studied the existence and uniqueness of solutions in $\left(W_{p}^{2,1}\left(Q_{\tau}\right)\right)^{2} \times L_{q}\left(0, \tau ; W_{q}^{2}(\Omega)\right)$, when $L$ is not a priori uniformly positive definite. In this paper we consider an energy transport model in which $L$ is not uniformly positive definite.

The Lyumkis model [3] is a typical energy transport model derived by physicists. In one space dimension we can write it directly as

$$
\left\{\begin{array}{l}
n_{t}+\left(j_{1}\right)_{x}=0 \\
\frac{3}{2}(n T)_{t}+\left(j_{2}\right)_{x}=V_{x} j_{1}+W \\
\lambda^{2} V_{x x}=n-C(x) \\
j_{1}=-\frac{2 \mu_{0}}{\sqrt{\pi}}\left(\left(n T^{\frac{1}{2}}\right)_{x}-\frac{n}{T^{\frac{1}{2}}} V_{x}\right) \\
j_{2}=-\frac{4 \mu_{0}}{\sqrt{\pi}}\left(\left(n T^{\frac{3}{2}}\right)_{x}-n T^{\frac{1}{2}} V_{x}\right) \\
W=\frac{2}{\sqrt{\pi}} \frac{n\left(T_{0}-T\right)}{\tau_{0} T^{\frac{1}{2}}}
\end{array}\right.
$$


in a parabolic domain $Q_{\tau}=(0,1) \times(0, \tau]$, where $\mu_{0}$ is the mobility constant, and $\tau_{0}$ is the relaxation time. System (1.4) is a special case of (1.1) (1.2), with coefficient matrix

$$
L=\frac{2 \mu_{0}}{\sqrt{\pi}} n T^{\frac{1}{2}}\left(\begin{array}{cc}
1 & 2 T \\
2 T & 6 T^{2}
\end{array}\right)
$$

Without loss of generality, we may choose $\frac{2 \mu_{0}}{\sqrt{\pi}}=\frac{4}{3 \sqrt{\pi} \tau_{0}}=\lambda=1$ to get

$$
\left\{\begin{array}{l}
n_{t}-\left(n T^{\frac{1}{2}}\right)_{x x}+\left(\frac{n}{T^{\frac{1}{2}}} V_{x}\right)_{x}=0 \\
n T_{t}-\frac{1}{3}\left(\left(n T^{\frac{3}{2}}\right)_{x x}-\left(n T^{\frac{1}{2}} V_{x}\right)_{x}\right)-\left(T^{\frac{1}{2}} T_{x} n\right)_{x} \\
\quad=\left(T_{x}-\frac{2}{3} V_{x}\right)\left(\left(n T^{\frac{1}{2}}\right)_{x}-\frac{n}{T^{\frac{1}{2}}} V_{x}\right)+\frac{n\left(T_{0}-T\right)}{T^{\frac{1}{2}}} \\
V_{x x}=n-C(x) .
\end{array}\right.
$$

There are other models simulating semiconductor devices, namely hydrodynamic models (HD) and drift diffusion models (DD). One can find a discussion on how these are related in [13]. In [10], L. Hsiao and T. Yang have investigated the relation between HD and DD models by comparing their large time behavior. Since ET and DD models can be obtained from the HD model under different scaling, one may expect that solutions derived in the context of the ET model will exhibit similar large time behavior as those for the HD model.

The main purpose of this paper is to study the global existence and the large time behavior of solutions to (1.4) in one space dimension when the initial data are close to a stationary solution of the corresponding solutions for the linear DD model. This provides, in a certain sense, a description of the relation of these models.

We consider the following initial conditions

$$
n(x, 0)=n_{I}(x), \quad T(x, 0)=T_{I}(x),
$$

and boundary conditions of insulation,

$$
j_{1}(0, t)=j_{1}(1, t)=0, \quad j_{2}(0, t)=j_{2}(1, t)=0, \quad V_{x}(0, t)=V_{x}(1, t)=0,
$$

which are equivalent to the boundary conditions

$$
n_{x}(0, t)=n_{x}(1, t)=0, \quad T_{x}(0, t)=T_{x}(1, t)=0, \quad V_{x}(0, t)=V_{x}(1, t)=0 .
$$

When one considers the solutions with $n>0, T>0$, it is required for compatibility that

$$
n_{I x}(0)=n_{I x}(1)=0, \quad T_{I x}(0)=T_{I x}(1)=0 .
$$

The special stationary problem we consider here is

$$
\begin{aligned}
& \left(\mathcal{N} T_{0}^{\frac{1}{2}}\right)_{x}-\frac{\mathcal{N}}{T_{0}^{\frac{1}{2}}} \mathcal{V}_{x}=0, \\
& \mathcal{V}_{x x}=\mathcal{N}-C(x),
\end{aligned}
$$

with boundary condition

$$
\mathcal{V}_{x}(0)=\mathcal{V}_{x}(1)=0
$$


Equations (1.11) and (1.12) are equivalent to those governing a linear drift diffusion model

with boundary conditions

$$
\left\{\begin{array}{l}
\left(T_{0} \mathcal{N}_{x}-\mathcal{N} \mathcal{V}_{x}\right)_{x}=0 \\
\mathcal{V}_{x x}=\mathcal{N}-C(x)
\end{array}\right.
$$

$$
\left\{\begin{array}{l}
\left(T_{0} \mathcal{N}_{x}-\mathcal{N} \mathcal{V}_{x}\right)(0)=0 \\
\mathcal{V}_{x}(0)=\mathcal{V}_{x}(1)=0
\end{array}\right.
$$

The following theorem is similar to one obtained in [10].

Theorem 1.1. Suppose $0<\underline{C} \leq C(x) \leq \bar{C}$ and let $(\mathcal{N}, \mathcal{V})$ be the solution of $(1.11)$ (1.12). Then

$$
\begin{gathered}
\underline{C} \leq \mathcal{N}(x) \leq \bar{C}, \quad x \in[0,1], \\
\left|\mathcal{N}_{x}(x)\right| \leq \frac{\bar{C}}{T_{0}}(\bar{C}-\underline{C}), \quad x \in[0,1], \\
\left|\mathcal{N}_{x x}(x)\right| \leq \frac{\bar{C} T_{0}+(\bar{C}-\underline{C}) \bar{C}}{T_{0}^{2}}(\bar{C}-\underline{C}), \quad x \in[0,1], \\
\left|\mathcal{V}_{x}(x)\right|,\left|\mathcal{V}_{x x}(x)\right| \leq \bar{C}-\underline{C}, \quad x \in[0,1] .
\end{gathered}
$$

The main theorem we obtain in this paper is

Theorem 1.2. Assume $V_{x}(x .0)-\mathcal{V}_{x}(x) \in H^{4}((0,1)), T_{I}(x) \in H^{3}((0,1)), \frac{\bar{C}}{2 T_{0}}+\frac{T_{0}}{3 \underline{C}}<$ $\sqrt{6}$. Then there exists a positive constant $\delta_{0}$ such that when

$$
(\bar{C}-\underline{C})+\left\|V_{x}(x, 0)-\mathcal{V}_{x}(x)\right\|_{H^{4}}+\left\|T_{I}(x)-T_{0}\right\|_{H^{3}} \leq \delta_{0},
$$

the problem $(1.6)(1.7)(1.9)$ has a unique solution $(n, T, V)$ in $(0,1) \times(0, \infty)$ satisfying

$$
\|n(\cdot, t)-\mathcal{N}(\cdot)\|_{H^{3}}+\left\|V_{x}(\cdot, t)-\mathcal{V}_{x}(\cdot)\right\|_{H^{4}}+\left\|T(\cdot, t)-T_{0}\right\|_{H^{3}} \leq C \exp (-\alpha t),
$$

for some positive constants $C$ and $\alpha$.

REMARK 1.1. In the theorem, $V_{x}(x, 0)$ is determined by $n_{I}(x)$ and $(1.6)_{3}$.

REMARK 1.2. The assumption $\frac{\bar{C}}{2 T_{0}}+\frac{T_{0}}{3 \underline{C}}<\sqrt{6}$ in the theorem ensures that the system is strongly parabolic. In that case, there exists a positive constant $0<\lambda<1$, depending on $\bar{C}, \underline{C}$, and $T_{0}$, such that

$$
\frac{\bar{C}}{2 T_{0}}+\frac{T_{0}}{3 \underline{C}}=2 \sqrt{(1-\lambda)\left(\frac{3}{2}-\lambda\right)}
$$

In fact, set $H(\mu)=4(1-\mu)\left(\frac{3}{2}-\mu\right)-\left(\frac{\bar{C}}{2 T_{0}}+\frac{T_{0}}{3 \underline{C}}\right)^{2}$. It is easy to show that there exists $\lambda, 0<\lambda<1$, such that $H(\lambda)=0$, provided $\frac{\bar{C}}{2 T_{0}}+\frac{T_{0}}{3 \underline{C}}<\sqrt{6}$. It is obvious that the set of $\bar{C}, \underline{C}$, and $T_{0}$ which satisfy $\frac{\bar{C}}{2 T_{0}}+\frac{T_{0}}{3 \underline{C}}<\sqrt{6}$ is nonempty. This guarantees that (1.17) is well posed. By (1.17), we have

$$
u^{2}-\left(\frac{\bar{C}}{2 T_{0}}+\frac{T_{0}}{3 \underline{C}}\right)|u||v|+\frac{3}{2} v^{2} \geq \lambda\left(u^{2}+v^{2}\right) .
$$


REMARK 1.3. In fact, in the problem discussed here, the asymptotic estimate can be obtained in $H^{1}$. However, an $H^{3}$ estimate must be proved in order to guarantee local existence. We will obtain these estimates in Sec. 3 .

2. Local existence and uniqueness. In this section, we discuss the local existence of the solution. Problem (1.6)(1.7)(1.9) can be written explicitly in the following form:

$$
\begin{aligned}
& n_{t}-T^{\frac{1}{2}} n_{x x}-\frac{n}{2 T^{\frac{1}{2}}} T_{x x}-\frac{1}{T^{\frac{1}{2}}} n_{x} T_{x}+\frac{n}{4 T^{\frac{3}{2}}} T_{x}^{2}+\frac{1}{T^{\frac{1}{2}}} n_{x} V_{x} \\
& -\frac{n}{2 T^{\frac{3}{2}}} T_{x} V_{x}+\frac{n}{T^{\frac{1}{2}}}(n-C)=0 \text {, } \\
& T_{t}-\frac{T^{\frac{3}{2}}}{3 n} n_{x x}-\frac{3}{2} T^{\frac{1}{2}} T_{x x}-\frac{3 T^{\frac{1}{2}}}{n} T_{x} n_{x}-\frac{5}{4 T^{\frac{1}{2}}} T_{x}^{2}+\frac{T^{\frac{1}{2}}}{n} n_{x} V_{x} \\
& +\frac{3}{2 T^{\frac{1}{2}}} T_{x} V_{x}-\frac{2}{3 T^{\frac{1}{2}}} V_{x}^{2}+\frac{T^{\frac{1}{2}}}{3}(n-C)+\frac{T-T_{0}}{T^{\frac{1}{2}}}=0, \\
& V_{x x}=n-C(x) \text {, } \\
& \left.n\right|_{t=0}=n_{I},\left.\quad T\right|_{t=0}=T_{I} \\
& n_{x}(0, t)=n_{x}(1, t)=T_{x}(0, t)=T_{x}(1, t)=V_{x}(0, t)=V_{x}(1, t)=0 \text {. }
\end{aligned}
$$

Local existence is established by

Theorem 2.1. Assume $0<2 \underline{D} \leq n_{I} \leq \frac{1}{2} \bar{D}, 0<2 \underline{T} \leq T_{I} \leq \frac{1}{2} \bar{T}$ and $\frac{n_{I}}{2 T_{I}}+\frac{T_{I}}{3 n_{I}}<\sqrt{6}$, $n_{I}, T_{I} \in H^{3}((0,1))$ with $\|n, T\|_{H^{3}((0,1))} \leq M_{0}$. Then there exists a $\tau>0$ such that the problem (2.1) has a unique solution $(n, T)$ which satisfies

$$
\sup _{0 \leq t \leq \tau}\left(\|n, T\|_{H^{3}((0,1))}+\left\|n_{t}, T_{t}\right\|_{H^{1}((0,1))}\right) \leq M<\infty .
$$

Proof. We will use the Banach fixed point theorem. We consider the space

$$
\begin{gathered}
\mathcal{X}=\left\{(n, T): \sup _{0 \leq t \leq \tau}\left(\|n, T\|_{H^{3}((0,1))}+\left\|n_{t}, T_{t}\right\|_{H^{1}((0,1))}\right) \leq M, M \geq M_{0},\right. \\
\left.0<\underline{D} \leq n \leq \bar{D}, 0<\underline{T} \leq T \leq \bar{T}, \frac{n}{2 T}+\frac{T}{3 n}<\sqrt{6}\right\}
\end{gathered}
$$

where $(0, \tau)$ is a time interval to be fixed later. It is easy to see that the following metric is complete in $\mathcal{X}$ :

$$
\||(n, T)|\|=\left[\sup _{0 \leq t \leq \tau}\left\{\|n(\cdot, t)\|_{L_{2}}^{2}+\|T(\cdot, t)\|_{L_{2}}^{2}\right\}+\int_{0}^{\tau}\left\{\left\|n_{x}(\cdot, t)\right\|_{L_{2}}^{2}+\left\|T_{x}(\cdot, t)\right\|_{L_{2}}^{2}\right\} d t\right]^{\frac{1}{2}}
$$

Define a map $F(u, v)=(n, T)$, for $(u, v) \in \mathcal{X}$, in the following way. First, for $u$ given above, solve the Poisson equation for $V$,

$$
\begin{aligned}
& V_{x x}=u-C(x), \quad \text { in }(0,1) \\
& V_{x}(0, t)=V_{x}(1, t)=0,
\end{aligned}
$$


and then solve the following linear system for $(n, T)$,

$$
\begin{aligned}
& n_{t}-v^{\frac{1}{2}} n_{x x}-\frac{u}{2 v^{\frac{1}{2}}} T_{x x}-\left(\frac{1}{v^{\frac{1}{2}}} v_{x}-\frac{V_{x}}{v^{\frac{1}{2}}}\right) n_{x}+\left(\frac{u v_{x}}{4 v^{\frac{3}{2}}}-\frac{u}{2 v^{\frac{3}{2}}} V_{x}\right) T_{x}+\frac{u}{v^{\frac{1}{2}}}(n-C)=0, \\
& T_{t}-\frac{v^{\frac{3}{2}}}{3 u} n_{x x}-\frac{3}{2} v^{\frac{1}{2}} T_{x x}-\left(\frac{3 v^{\frac{1}{2}}}{u} u_{x}+\frac{5 v_{x}}{4 v^{\frac{1}{2}}}-\frac{3}{2 v^{\frac{1}{2}}} V_{x}\right) T_{x} \\
& +\frac{v^{\frac{1}{2}}}{u} V_{x} n_{x}+\frac{v^{\frac{1}{2}}}{3}(n-C)-\frac{2}{3 v^{\frac{1}{2}}} V_{x}^{2}+\frac{T-T_{0}}{v^{\frac{1}{2}}}=0, \\
& \left.n\right|_{t=0}=n_{I},\left.\quad T\right|_{t=0}=T_{I} \\
& n_{x}(0, t)=n_{x}(1, t)=T_{x}(0, t)=T_{x}(1, t)=0 \text {. }
\end{aligned}
$$

By $(u, v) \in \mathcal{X}$ and the Sobolev embedding theorem, all the coefficients in the above strongly parabolic system belong to $L_{\infty}\left(Q_{\tau}\right)$ and there exists $\lambda_{1}>0$ such that

$$
\frac{u}{2 v}+\frac{v}{3 u} \leq 2 \sqrt{\left(1-\lambda_{1}\right)\left(\frac{3}{2}-\lambda_{1}\right)} \text {. }
$$

Thus, by the classical theory for linear parabolic systems, (2.5) is solvable.

Now one has to show that $F$ maps $\mathcal{X}$ into itself and $F$ is contractive in $\mathcal{X}$, provided $\tau$ is sufficiently small.

In the sequel, $K$ will denote a generic constant which depends on $M, \underline{D}, \underline{T}$, and $\lambda_{1}>0$.

By (2.4), we have

$$
\left|V_{x}\right|,\left|V_{x t}\right| \leq K
$$

Multiplying $(2.5)_{1}$ by $n$, and integrating over $[0,1]$, we deduce

$$
\frac{1}{2} \frac{d}{d t} \int_{0}^{1} n^{2}+\int_{0}^{1}\left(v^{\frac{1}{2}} n_{x}^{2}+\frac{u}{2 v^{\frac{1}{2}}} n_{x} T_{x}\right) \leq \varepsilon \int_{0}^{1}\left(n_{x}^{2}+T_{x}^{2}\right)+K \int_{0}^{1}\left(n^{2}+T^{2}\right)+K .
$$

Multiplying $(2.5)_{2}$ by $T$, and integrating over [0,1], yields

$$
\frac{1}{2} \frac{d}{d t} \int_{0}^{1} T^{2}+\int_{0}^{1}\left(\frac{v^{\frac{3}{2}}}{3 u} n_{x} T_{x}+\frac{3}{2} v^{\frac{1}{2}} T_{x}^{2}\right) \leq \varepsilon \int_{0}^{1}\left(n_{x}^{2}+T_{x}^{2}\right)+K \int_{0}^{1}\left(n^{2}+T^{2}\right)+K .
$$

Combining (2.8) with (2.9), using (2.6), and choosing $\varepsilon=\frac{T \lambda_{1}}{2}$, we obtain

$$
\frac{d}{d t} \int_{0}^{1}\left(n^{2}+T^{2}\right)+\underline{T} \lambda_{1} \int_{0}^{1}\left(n_{x}^{2}+T_{x}^{2}\right) \leq K \int_{0}^{1}\left(n^{2}+T^{2}\right)+K .
$$

Differentiating $(2.5)_{1}$ and $(2.5)_{2}$ with respect to $x$, multiplying them by $n_{x}$ and $T_{x}$, respectively, and integrating by parts, whenever necessary, we deduce

$$
\frac{d}{d t} \int_{0}^{1}\left(n_{x}^{2}+T_{x}^{2}\right)+\underline{T} \lambda_{1} \int_{0}^{1}\left(n_{x x}^{2}+T_{x x}^{2}\right) \leq K \int_{0}^{1}\left(n_{x}^{2}+T_{x}^{2}+n^{2}+T^{2}\right)+K,
$$

where we have used again (2.6).

Adding (2.11) to (2.10), applying Gronwall's inequality and integrating over $[0, \tau]$,

$$
\sup _{0 \leq t \leq \tau} \int_{0}^{1}\left(n_{x}^{2}+T_{x}^{2}+n^{2}+T^{2}\right)+\int_{0}^{\tau} \int_{0}^{1}\left(n_{x x}^{2}+T_{x x}^{2}+n_{x}^{2}+T_{x}^{2}\right) \leq \tau M_{0} K e^{K \tau} .
$$


Thus if we choose $\tau_{1}$ sufficiently small,

$$
\sup _{0 \leq t \leq \tau_{1}}\left(\|n\|_{H^{1}((0,1))}^{2}+\|T\|_{H^{1}((0,1))}^{2}\right)+\int_{0}^{\tau_{1}}\left(\|n(\cdot, t)\|_{H^{2}((0,1))}^{2}+\|T(\cdot, t)\|_{H^{2}((0,1))}^{2}\right) \leq M .
$$

The above inequality shows that both $n_{x}$ and $T_{x}$ lie in $V_{2}\left(Q_{\tau_{1}}\right)$, which implies, by the Sobolev embedding theorem, that both $n_{x}$ and $T_{x}$ are in $L_{\infty}\left(Q_{\tau_{1}}\right)$, i.e.,

$$
\left|n_{x}\right|,\left|T_{x}\right| \leq K
$$

The next step is to estimate $n_{x x}$ and $T_{x x}$. To that end, it is not possible to follow the earlier procedure by differentiating (2.5) twice with respect to $x$ and multiplying by $n_{x x}$ or $T_{x x}$, since we don't have appropriate boundary conditions to allow for integration by parts. Fortunately, we can estimate $n_{t}$ and $T_{t}$ in the place of $n_{x x}$ and $T_{x x}$ with the help of the equations $(2.5)_{1,2}$ themselves. By the boundary conditions of $(2.5)$, we have

$$
n_{x t}(0, t)=n_{x t}(1, t)=T_{x t}(0, t)=T_{x t}(1, t)=0 .
$$

Differentiating $(2.5)_{1}$ with respect to $t$, multiplying by $n_{t}$, and integrating over $[0,1]$, we obtain

$$
\frac{1}{2} \frac{d}{d t} \int_{0}^{1} n_{t}^{2}-\int_{0}^{1}\left(v^{\frac{1}{2}} n_{x x}\right)_{t} n_{t}-\int_{0}^{1}\left(\frac{u}{2 v^{\frac{1}{2}}} T_{x x}\right)_{t} n_{t}=G_{1}
$$

where by $(u, v) \in \mathcal{X}$ and $(2.7),(2.14)$,

$$
\begin{aligned}
G_{1} & =\int_{0}^{1}\left[\left(\frac{1}{v^{\frac{1}{2}}} v_{x}-\frac{V_{x}}{v^{\frac{1}{2}}}\right) n_{x}\right]_{t} n_{t}-\left[\left(\frac{u v_{x}}{4 v^{\frac{3}{2}}}-\frac{u}{2 v^{\frac{3}{2}}} V_{x}\right) T_{x}\right]_{t} n_{t}-\left[\frac{u}{v^{\frac{1}{2}}}(n-C)\right]_{t} n_{t} \\
& \leq \varepsilon \int_{0}^{1}\left(n_{x t}^{2}+T_{x t}^{2}\right)+K \int_{0}^{1}\left(n_{x x}^{2}+T_{x x}^{2}+n_{t}^{2}+T_{t}^{2}\right)+K .
\end{aligned}
$$

On the other hand,

$$
\begin{aligned}
& -\int_{0}^{1}\left(v^{\frac{1}{2}} n_{x x}\right)_{t} n_{t}-\int_{0}^{1}\left(\frac{u}{2 v^{\frac{1}{2}}} T_{x x}\right)_{t} n_{t} \\
= & \int_{0}^{1} v^{\frac{1}{2}} n_{x t}^{2}+\int_{0}^{1} \frac{u}{2 v^{\frac{1}{2}}} T_{x t} n_{x t}+\int_{0}^{1}\left(v^{\frac{1}{2}}\right)_{t} n_{x} n_{x t}+\int_{0}^{1}\left(v^{\frac{1}{2}}\right)_{x t} n_{x} n_{t}+\int_{0}^{1}\left(v^{\frac{1}{2}}\right)_{x} n_{x t} n_{t} \\
& +\int_{0}^{1}\left(\frac{u}{2 v^{\frac{1}{2}}}\right)_{t} T_{x} n_{x t}+\int_{0}^{1}\left(\frac{u}{2 v^{\frac{1}{2}}}\right)_{x t} T_{x} n_{t}+\int_{0}^{1}\left(\frac{u}{2 v^{\frac{1}{2}}}\right)_{x} T_{x t} n_{t} .
\end{aligned}
$$

Combining (2.16), (2.17), and (2.18), we deduce

$\frac{1}{2} \frac{d}{d t} \int_{0}^{1} n_{t}^{2}+\int_{0}^{1}\left(v^{\frac{1}{2}} n_{x t}^{2}+\frac{u}{2 v^{\frac{1}{2}}} T_{x t} n_{x t}\right) \leq \varepsilon \int_{0}^{1}\left(n_{x t}^{2}+T_{x t}^{2}\right)+K \int_{0}^{1}\left(n_{x x}^{2}+T_{x x}^{2}+n_{t}^{2}+T_{t}^{2}\right)+K$.

Similarly, differentiating $(2.5)_{1}$ with respect to $t$, multiplying by $n_{t}$, and integrating over $[0,1]$ yields

$\frac{1}{2} \frac{d}{d t} \int_{0}^{1} T_{t}^{2}+\int_{0}^{1}\left(\frac{v^{\frac{3}{2}}}{3 u} n_{x t} T_{x t}+\frac{3}{2} v^{\frac{1}{2}} T_{x t}^{2}\right) \leq \varepsilon \int_{0}^{1}\left(n_{x t}^{2}+T_{x t}^{2}\right)+K \int_{0}^{1}\left(n_{x x}^{2}+T_{x x}^{2}+n_{t}^{2}+T_{t}^{2}\right)+K$. 
Again, combining (2.6), (2.19), and (2.20), and choosing $\varepsilon=\frac{T \lambda_{1}}{2}$, we obtain

$$
\frac{1}{2} \frac{d}{d t} \int_{0}^{1}\left(n_{t}^{2}+T_{t}^{2}\right)+\int_{0}^{1}\left(n_{x t}^{2}+T_{x t}^{2}\right) \leq K \int_{0}^{1}\left(n_{x x}^{2}+T_{x x}^{2}+n_{t}^{2}+T_{t}^{2}\right)+K .
$$

We can find the relation between $n_{x x}, T_{x x}$ and $n_{t}, T_{t}$ by direct calculation, with the help of $(2.5)_{1,2}$,

$$
\begin{aligned}
& n_{x x}=\frac{9}{8 v^{\frac{1}{2}}} n_{t}-\frac{3 u}{8 v^{\frac{3}{2}}} T_{t}+\frac{9}{8 v^{\frac{1}{2}}} H_{1}-\frac{3 u}{8 v^{\frac{3}{2}}} H_{2}, \\
& T_{x x}=-\frac{v^{\frac{1}{2}}}{4 u} n_{t}+\frac{3}{4 v^{\frac{1}{2}}} T_{t}-\frac{v^{\frac{1}{2}}}{4 u} H_{1}+\frac{3}{4 v^{\frac{1}{2}}} H_{2}, \\
& H_{1}=-\left(\frac{1}{v^{\frac{1}{2}}} v_{x}-\frac{V_{x}}{v^{\frac{1}{2}}}\right) n_{x}+\left(\frac{u v_{x}}{4 v^{\frac{3}{2}}}-\frac{u}{2 v^{\frac{3}{2}}} V_{x}\right) T_{x}+\frac{u}{v^{\frac{1}{2}}}(n-C), \\
& H_{2}=-\left(\frac{3 v^{\frac{1}{2}}}{u} u_{x}+\frac{5 v_{x}}{4 v^{\frac{1}{2}}}-\frac{3}{2 v^{\frac{1}{2}}} V_{x}\right) T_{x}+\frac{v^{\frac{1}{2}}}{u} V_{x} n_{x}+\frac{v^{\frac{1}{2}}}{3}(n-C)-\frac{2}{3 v^{\frac{1}{2}}} V_{x}^{2}+\frac{T-T_{0}}{v^{\frac{1}{2}}} .
\end{aligned}
$$

Thus, (2.21) implies

$$
\frac{1}{2} \frac{d}{d t} \int_{0}^{1}\left(n_{t}^{2}+T_{t}^{2}\right)+\int_{0}^{1}\left(n_{x t}^{2}+T_{x t}^{2}\right) \leq K \int_{0}^{1}\left(n_{t}^{2}+T_{t}^{2}\right)+K .
$$

Differentiating $(2.5)_{1,2}$ with respect to $x$, and then with respect to $t$, multiplying by $n_{x t}$ and $T_{x t}$, respectively, we get with the help of (2.15),

$$
\begin{aligned}
& \frac{1}{2} \frac{d}{d t} \int_{0}^{1} n_{x t}^{2}+\int_{0}^{1}\left(v^{\frac{1}{2}} n_{x x t}^{2}+\frac{u}{2 v^{\frac{1}{2}}} T_{x x t} n_{x x t}\right)+\int_{0}^{1}\left(v^{\frac{1}{2}}\right)_{t} n_{x x} n_{x x t} \\
& \quad+\int_{0}^{1}\left(\frac{u}{2 v^{\frac{1}{2}}}\right)_{t} T_{x x} n_{x x t}=G_{2} \\
& \frac{1}{2} \frac{d}{d t} \int_{0}^{1} T_{x t}^{2}+\int_{0}^{1}\left(\frac{3}{2} v^{\frac{1}{2}} T_{x x t}^{2}+\frac{v^{\frac{3}{2}}}{3 u} T_{x x t} n_{x x t}\right)+\int_{0}^{1}\left(\frac{3}{2} v^{\frac{1}{2}}\right)_{t} T_{x x} T_{x x t} \\
& \quad+\int_{0}^{1}\left(\frac{v^{\frac{3}{2}}}{3 u}\right)_{t} n_{x x} T_{x x t}=G_{3},
\end{aligned}
$$

where, by virtue of $(u, v) \in \mathcal{X},(2.7)$, and (2.14),

$$
\begin{aligned}
G_{2}= & -\int_{0}^{1}\left[\left(\frac{1}{v^{\frac{1}{2}}} v_{x}-\frac{V_{x}}{v^{\frac{1}{2}}}\right) n_{x}\right]_{t} n_{x x t}+\int_{0}^{1}\left[\left(\frac{u v_{x}}{4 v^{\frac{3}{2}}}-\frac{u}{2 v^{\frac{3}{2}}} V_{x}\right) T_{x}\right]_{t} n_{x x t} \\
& +\int_{0}^{1}\left[\frac{u}{v^{\frac{1}{2}}}(n-C)\right]_{t} n_{x x t} \\
\leq & \varepsilon \int_{0}^{1} n_{x x t}^{2}+K \int_{0}^{1}\left(n_{x t}^{2}+T_{x t}^{2}+n_{x x}^{2}+T_{x x}^{2}+n_{t}^{2}+T_{t}^{2}\right)+K, \\
G_{3}= & -\int_{0}^{1}\left[\left(\frac{3 v^{\frac{1}{2}}}{u} u_{x}+\frac{5 v_{x}}{4 v^{\frac{1}{2}}}-\frac{3 V_{x}}{2 v^{\frac{1}{2}}}\right) T_{x}\right]_{t} T_{x x t}+\int_{0}^{1}\left[\frac{v^{\frac{1}{2}}}{u} V_{x} n_{x}-\frac{2}{3 v^{\frac{1}{2}}} V_{x}^{2}\right]_{t} T_{x x t} \\
& +\int_{0}^{1}\left[\frac{v^{\frac{1}{2}}}{3}(n-C)+\frac{T-T_{0}}{v^{\frac{1}{2}}}\right]_{t} T_{x x t} \\
\leq & \varepsilon \int_{0}^{1} T_{x x t}^{2}+K \int_{0}^{1}\left(n_{x t}^{2}+T_{x t}^{2}+n_{x x}^{2}+T_{x x}^{2}+n_{t}^{2}+T_{t}^{2}\right)+K .
\end{aligned}
$$


Thus, since $(u, v) \in \mathcal{X}$, and by account of $(2.22)$,

$$
\frac{d}{d t} \int_{0}^{1}\left(n_{x t}^{2}+T_{x t}^{2}\right)+\int_{0}^{1}\left(n_{x x t}^{2}+T_{x x t}^{2}\right) \leq K \int_{0}^{1}\left(n_{x t}^{2}+T_{x t}^{2}+n_{t}^{2}+T_{t}^{2}\right)+K
$$

Similar to (2.12) and (2.13), by Gronwall's inequality, we can choose $\tau_{2} \leq \tau_{1}$ so that

$$
\sup _{0 \leq t \leq \tau_{2}}\left(\left\|n_{t}\right\|_{H^{1}((0,1))}^{2}+\left\|T_{t}\right\|_{H^{1}((0,1))}^{2}\right)+\int_{0}^{\tau_{2}}\left(\left\|n_{t}(\cdot, t)\right\|_{H^{2}((0,1))}^{2}+\left\|T_{t}(\cdot, t)\right\|_{H^{2}((0,1))}^{2}\right) \leq M .
$$

By the estimates (2.14) and (2.27), we have $(n, T) \in C^{1, \frac{1}{2}}\left(Q_{\tau_{2}}\right)$. Notice that the initial data satisfy $0<2 \underline{D} \leq n_{I} \leq \frac{1}{2} \bar{D}, 0<2 \underline{T} \leq T_{I} \leq \frac{1}{2} \bar{T}, \frac{n_{I}}{2 T_{I}}+\frac{T_{I}}{3 n_{I}}<\sqrt{6}$. Hence there exists $\tau_{3} \leq \tau_{2}$ such that

$$
0<\underline{D} \leq n \leq \bar{D}, 0<\underline{T} \leq T \leq \bar{T}, \frac{n}{2 T}+\frac{T}{3 n}<\sqrt{6}, \quad \forall t \in\left[0, \tau_{3}\right]
$$

Thus we have obtained that $(n, T) \in \mathcal{X}$.

Now we show that the map $F$ is contractive on a sufficiently small time interval $\left(0, \tau_{4}\right)$ with $\tau_{4} \leq \tau_{3}$. For the difference

$$
(\delta n, \delta T)=\left(n_{1}, T_{1}\right)-\left(n_{2}, T_{2}\right)=F\left(u_{1}, v_{1}\right)-F\left(u_{2}, v_{2}\right)
$$

we obtain the following initial boundary problem:

$$
\begin{aligned}
(\delta n)_{t} & -\sqrt{v_{1}}(\delta n)_{x x}-\frac{u_{1}}{2 \sqrt{v_{1}}}(\delta T)_{x x}-\left(\frac{v_{1 x}}{\sqrt{v_{1}}}-\frac{V_{1 x}}{\sqrt{v_{1}}}\right)(\delta n)_{x}+\left(\frac{u_{1} v_{1 x}}{4 \sqrt{v_{1}^{3}}}-\frac{u_{1} V_{1 x}}{2 \sqrt{v_{1}^{3}}}\right)(\delta T)_{x} \\
& +\frac{u_{1}}{\sqrt{v_{1}}}(\delta n)+\left(\frac{n_{2 x}}{\sqrt{v_{1}}}-\frac{u_{1} T_{2 x}}{2 \sqrt{v_{1}^{3}}}\right)(\delta V)_{x}+\left(-\frac{n_{2 x}}{\sqrt{v_{1}}}+\frac{u_{1} T_{2 x}}{4 \sqrt{v_{1}^{3}}}\right)(\delta v)_{x}-\frac{T_{2 x x}}{2 \sqrt{v_{1}}}(\delta u) \\
& -\left(\frac{n_{2 x x}}{\sqrt{v_{1}}+\sqrt{v_{2}}}-\frac{u_{2 x x}}{2 \sqrt{v_{1} v_{2}}\left(\sqrt{v_{1}}+\sqrt{v_{2}}\right)}\right)(\delta v)+f_{1}(\delta u)+f_{2}(\delta v)=0, \\
(\delta T)_{t}- & \frac{\sqrt{v_{1}^{3}}}{3 u_{1}}(\delta n)_{x x}-\frac{3 \sqrt{v_{1}}}{2}(\delta T)_{x x}+\frac{\sqrt{v_{1}} V_{1 x}}{u_{1}}(\delta n)_{x} \\
& -\left(\frac{3 \sqrt{v_{1}}}{u_{1}} u_{1 x}+\frac{5 v_{1 x}}{4 \sqrt{v_{1}}}-\frac{3}{2 \sqrt{v_{1}}} V_{1 x}\right)(\delta T)_{x} \\
& +\frac{\sqrt{v_{1}}}{3}(\delta n)+\frac{1}{\sqrt{v_{1}}}(\delta T)+f_{3}(\delta V)_{x}-\frac{3 \sqrt{v_{1}} T_{2 x}}{u_{1}}(\delta u)_{x}-\frac{5 T_{2 x}}{4 \sqrt{v_{1}}}(\delta v)_{x} \\
& -\left[\frac{1}{3 u_{1}}\left(\sqrt{v_{1}}+\frac{v_{2}}{\sqrt{v_{1}}+\sqrt{v_{2}}}\right) n_{2 x x}+\frac{3}{2} \frac{T_{2 x x}}{\sqrt{v_{1}}+\sqrt{v_{2}}}\right](\delta v) \\
+ & \frac{\sqrt{v_{2}^{3}}}{3 u_{1} u_{2}} n_{2 x x}(\delta u)+f_{4}(\delta u)+f_{5}(\delta v)=0 \\
\left.(\delta n)\right|_{t=} & =\left.(\delta T)\right|_{t=0}=0, \quad(\delta n)_{x}(0, t)=(\delta n)_{x}(1, t)=(\delta T)_{x}(0, t)=(\delta T)_{x}(1, t)=0
\end{aligned}
$$


where $(\delta V)_{x x}=(\delta u),(\delta V)_{x}(0, t)=(\delta V)_{x}(1, t)=0, V_{i x x}=u_{i}-C(x), V_{i x}(0, t)=$ $V_{i x}(1, t)=0, i=1,2$, and

$$
\begin{aligned}
f_{1}= & \frac{v_{2 x} T_{2 x}}{4 \sqrt{v_{1}^{3}}}-\frac{V_{2 x} T_{2 x}}{2 \sqrt{v_{1}^{3}}}+\frac{1}{\sqrt{v_{1}}}\left(n_{2}-C(x)\right) \\
f_{2}= & \frac{v_{2 x} n_{2 x}}{\sqrt{v_{1} v_{2}}\left(\sqrt{v_{1}}+\sqrt{v_{2}}\right)}+\left(\frac{1}{v_{1} v_{2}\left(\sqrt{v_{1}}+\sqrt{v_{2}}\right)}-\frac{1}{\left.\sqrt{v_{1}^{3} v_{2}}-\frac{1}{v_{1} \sqrt{v_{2}^{3}}}\right)}\right. \\
& \left(\frac{u_{2} v_{2 x} T_{2 x}}{4}-\frac{u_{2} V_{2 x} T_{2 x}}{2}\right) \\
& -\frac{V_{2 x} n_{2 x}}{\sqrt{v_{1} v_{2}}\left(\sqrt{v_{1}}+\sqrt{v_{2}}\right)}-\frac{u_{2}}{\sqrt{v_{1} v_{2}}\left(\sqrt{v_{1}}+\sqrt{v_{2}}\right)}\left(n_{2}-C(x)\right), \\
f_{3}= & \frac{\sqrt{v_{1}} n_{2 x}}{u_{1}}+\frac{3 T_{2 x}}{2 \sqrt{v_{1}}}-\frac{2 V_{1 x}}{3 \sqrt{v_{1}}}-\frac{2 V_{2 x}}{3 \sqrt{v_{1}}}, \\
f_{4}= & \frac{3 \sqrt{v_{2}} u_{2 x} T_{2 x}}{u_{1} u_{2}}-\frac{\sqrt{v_{2}} V_{2 x} n_{2 x}}{u_{1} u_{2}}, \\
f_{5}= & -\frac{3 u_{2 x} T_{2 x}}{u_{1}\left(\sqrt{v_{1}}+\sqrt{v_{2}}\right)}+\frac{5 v_{2 x} T_{2 x}}{4 \sqrt{v_{1} v_{2}}\left(\sqrt{v_{1}}+\sqrt{v_{2}}\right)}+\frac{V_{2 x} n_{2 x}}{u_{1}\left(\sqrt{v_{1}}+\sqrt{v_{2}}\right)}-\frac{3 V_{2 x} T_{2 x}}{2 \sqrt{v_{1} v_{2}}\left(\sqrt{v_{1}}+\sqrt{v_{2}}\right)} \\
& +\frac{2 V_{2 x}^{2}}{3 \sqrt{v_{1} v_{2}}\left(\sqrt{v_{1}}+\sqrt{v_{2}}\right)}+\frac{n_{2}-C(x)}{3\left(\sqrt{v_{1}}+\sqrt{v_{2}}\right)}-\frac{T_{0}}{\sqrt{v_{1} v_{2}}\left(\sqrt{v_{1}}+\sqrt{v_{2}}\right)} .
\end{aligned}
$$

To deal with $(2.30)$, we need some preparation. We first note that

$$
\left|f_{1}\right|,\left|f_{2}\right|,\left|f_{3}\right|,\left|f_{4}\right|,\left|f_{5}\right| \leq K(M, \underline{D}, \underline{T})
$$

since $(u, v),(n, p) \in \mathcal{X}$. Notice that $(\delta V)_{x x}=(\delta u),(\delta V)_{x}(0, t)=(\delta V)_{x}(1, t)=0$. Hence we have $\left\|(\delta V)_{x}\right\|_{L_{2}\left(Q_{\tau}\right)} \leq K\|(\delta u)\|_{L_{2}\left(Q_{\tau}\right)}$.

Multiplying $(2.30)_{1}$ by $(\delta n)$ and integrating over $[0,1]$ yields

$$
\begin{aligned}
& \frac{1}{2} \frac{d}{d t} \int_{0}^{1}(\delta n)^{2}+\int_{0}^{1}\left(\sqrt{v_{1}}(\delta n)_{x}^{2}+\frac{u_{1}}{2 \sqrt{v_{1}}}(\delta T)_{x}(\delta n)_{x}\right) \\
& +\int_{0}^{1} \frac{T_{2 x}}{2 \sqrt{v_{1}}}\left((\delta u)_{x}(\delta n)+(\delta u)(\delta n)_{x}\right) \\
& +\int_{0}^{1}\left(\frac{n_{2 x}}{\sqrt{v_{1}}+\sqrt{v_{2}}}-\frac{u_{2} T_{2 x}}{2 \sqrt{v_{1} v_{2}}\left(\sqrt{v_{1}}+\sqrt{v_{2}}\right)}\right)\left((\delta v)_{x}(\delta n)+(\delta v)(\delta n)_{x}\right) \\
\leq & \varepsilon \int_{0}^{1}\left((\delta n)_{x}^{2}+(\delta T)_{x}^{2}\right)+K(M, \varepsilon) \int_{0}^{1}\left((\delta n)^{2}+(\delta T)^{2}\right) \\
& +\varepsilon \int_{0}^{1}\left((\delta u)_{x}^{2}+(\delta v)_{x}^{2}\right)+K(M, \varepsilon) \int_{0}^{1}\left((\delta u)^{2}+(\delta v)^{2}+(\delta V)_{x}^{2}\right) .
\end{aligned}
$$

It then follows

$$
\begin{aligned}
& \frac{1}{2} \frac{d}{d t} \int_{0}^{1}(\delta n)^{2}+\int_{0}^{1}\left(\sqrt{v_{1}}(\delta n)_{x}^{2}+\frac{u_{1}}{2 \sqrt{v_{1}}}(\delta T)_{x}(\delta n)_{x}\right) \\
\leq & \varepsilon \int_{0}^{1}\left((\delta n)_{x}^{2}+(\delta T)_{x}^{2}\right)+K(M, \varepsilon) \int_{0}^{1}\left((\delta n)^{2}+(\delta T)^{2}\right) \\
& +\varepsilon \int_{0}^{1}\left((\delta u)_{x}^{2}+(\delta v)_{x}^{2}\right)+K(M, \varepsilon) \int_{0}^{1}\left((\delta u)^{2}+(\delta v)^{2}\right) .
\end{aligned}
$$


Multiplying $(2.30)_{2}$ by $(\delta T)$ and integrating over $[0,1]$, we obtain

$$
\begin{aligned}
& \frac{1}{2} \frac{d}{d t} \int_{0}^{1}(\delta T)^{2}+\int_{0}^{1}\left(\frac{\sqrt{v_{1}^{3}}}{3 u_{1}}(\delta n)_{x}(\delta T)_{x}+\frac{3 \sqrt{v_{1}}}{2}(\delta T)_{x}^{2}\right) \\
& +\int_{0}^{1}\left[\frac{1}{3 u_{1}}\left(\sqrt{v_{1}}+\frac{v_{2}}{\sqrt{v_{1}}+\sqrt{v_{2}}}\right) n_{2 x}+\frac{T_{2 x}}{\sqrt{v_{1}}+\sqrt{v_{2}}}\right]\left((\delta v)_{x}(\delta T)+(\delta v)(\delta T)_{x}\right) \\
& -\int_{0}^{1} \frac{\sqrt{v_{2}^{3}}}{3 u_{1} u_{2}} n_{2 x}\left((\delta u)_{x}(\delta T)+(\delta u)(\delta T)_{x}\right) \\
\leq & \varepsilon \int_{0}^{1}\left((\delta n)_{x}^{2}+(\delta T)_{x}^{2}\right)+K(M, \varepsilon) \int_{0}^{1}\left((\delta n)^{2}+(\delta T)^{2}\right) \\
& +\varepsilon \int_{0}^{1}\left((\delta u)_{x}^{2}+(\delta v)_{x}^{2}\right)+K(M, \varepsilon) \int_{0}^{1}\left((\delta u)^{2}+(\delta v)^{2}+(\delta V)_{x}^{2}\right) .
\end{aligned}
$$

Similar to $(2.32)$, we have

$$
\begin{aligned}
& \frac{1}{2} \frac{d}{d t} \int_{0}^{1}(\delta T)^{2}+\int_{0}^{1}\left(\frac{\sqrt{v_{1}^{3}}}{3 u_{1}}(\delta n)_{x}(\delta T)_{x}+\frac{3 \sqrt{v_{1}}}{2}(\delta T)_{x}^{2}\right) \\
\leq & \varepsilon \int_{0}^{1}\left((\delta n)_{x}^{2}+(\delta T)_{x}^{2}\right)+K(M, \varepsilon) \int_{0}^{1}\left((\delta n)^{2}+(\delta T)^{2}\right) \\
& +\varepsilon \int_{0}^{1}\left((\delta u)_{x}^{2}+(\delta v)_{x}^{2}\right)+K(M, \varepsilon) \int_{0}^{1}\left((\delta u)^{2}+(\delta v)^{2}\right) .
\end{aligned}
$$

Since $\left(u_{1}, v_{1}\right) \in \mathcal{X}$, and $\frac{u_{1}}{2 v_{1}}+\frac{v_{1}}{3 u_{1}} \leq 2 \sqrt{\left(1-\lambda_{1}\right)\left(\frac{3}{2}-\lambda_{1}\right)}$,

$$
\begin{aligned}
& \sqrt{v_{1}}(\delta n)_{x}^{2}+\frac{u_{1}}{2 \sqrt{v_{1}}}(\delta T)_{x}(\delta n)_{x}+\frac{\sqrt{v_{1}^{3}}}{3 u_{1}}(\delta n)_{x}(\delta T)_{x}+\frac{3 \sqrt{v_{1}}}{2}(\delta T)_{x}^{2} \\
= & \sqrt{v_{1}}\left[(\delta n)_{x}^{2}+\left(\frac{u_{1}}{2 v_{1}}+\frac{v_{1}}{3 u_{1}}\right)(\delta n)_{x}(\delta T)_{x}+\frac{3 \sqrt{v_{1}}}{2}(\delta T)_{x}^{2}\right] \\
\geq & \sqrt{T} \lambda_{1}\left((\delta n)_{x}^{2}+(\delta T)_{x}^{2}\right) .
\end{aligned}
$$

Combining (2.34) with (2.32) and (2.33) yields

$$
\begin{aligned}
& \frac{d}{d t} \int_{0}^{1}\left((\delta n)^{2}+(\delta T)^{2}\right)+\int_{0}^{1}\left((\delta n)_{x}^{2}+(\delta T)_{x}^{2}\right) \\
\leq & K(M) \int_{0}^{1}\left((\delta n)^{2}+(\delta T)^{2}\right)+\varepsilon \int_{0}^{1}\left((\delta u)_{x}^{2}+(\delta v)_{x}^{2}\right)+K(M, \varepsilon) \int_{0}^{1}\left((\delta u)^{2}+(\delta v)^{2}\right) .
\end{aligned}
$$

By Gronwall's inequality,

$$
\int_{0}^{1}\left((\delta n)^{2}+(\delta T)^{2}\right) \leq\left(\varepsilon \int_{0}^{\tau} \int_{0}^{1}(\delta u)_{x}^{2}+(\delta v)_{x}^{2}+K(M, \varepsilon) \int_{0}^{\tau} \int_{0}^{1}(\delta u)^{2}+(\delta v)^{2}\right) e^{K(M) \tau}
$$

Thus

$$
\begin{aligned}
& \frac{d}{d t} \int_{0}^{1}\left((\delta n)^{2}+(\delta T)^{2}\right)+\int_{0}^{1}\left((\delta n)_{x}^{2}+(\delta T)_{x}^{2}\right) \\
\leq & \left(\varepsilon K(M) \int_{0}^{\tau} \int_{0}^{1}(\delta u)_{x}^{2}+(\delta v)_{x}^{2}+K(M, \varepsilon) \int_{0}^{\tau} \int_{0}^{1}(\delta u)^{2}+(\delta v)^{2}\right) e^{K(M) \tau} \\
& +\varepsilon \int_{0}^{1}\left((\delta u)_{x}^{2}+(\delta v)_{x}^{2}\right)+K(M, \varepsilon) \int_{0}^{1}\left((\delta u)^{2}+(\delta v)^{2}\right) .
\end{aligned}
$$


Integrating with respect to $t$, over $[0, \tau]$, and choosing $\varepsilon$ and $\tau_{4} \leq \tau_{3}$ sufficiently small, we conclude

$$
|\|(\delta n, \delta T)\|| \leq \frac{1}{2}|\|(\delta u, \delta v)\|| .
$$

Thus there exists precisely one fixed point $\left(n^{*}, T^{*}\right)$ with $\left(n^{*}, T^{*}\right)=F\left(n^{*}, T^{*}\right)$ in $\mathcal{X}$. This fixed point is the unique solution to $(2.1)$.

3. Asymptotic behavior. In this section we obtain the asymptotic estimate that proves Theorem 1.2. Set $\psi=V_{x}-\mathcal{V}_{x}, \varphi=n-\mathcal{N}, f=T-T_{0}$.

Lemma 3.1. There exist positive constants $\delta>0$ and $\beta>0$ such that, if

$$
\sup _{0 \leq t \leq \tau}\left(\|\psi(\cdot, t)\|_{H^{2}}+\|f(\cdot, t)\|_{H^{1}}\right) \leq \delta
$$

for all $\tau>0$, then

$$
\|\psi(\cdot, t)\|_{H^{2}}+\|f(\cdot, t)\|_{H^{1}} \leq C\left(\|\psi(\cdot, 0)\|_{H^{2}}+\|f(\cdot, 0)\|_{H^{1}}\right) \exp (-\beta t),
$$

for any $t \in[0, \tau]$.

Proof. From (1.9) and (1.12) we get

$$
\begin{gathered}
\psi(0, t)=\psi(1, t)=0, \\
\psi_{x x}(0, t)=\psi_{x x}(1, t)=0, \\
f_{x}(0, t)=f_{x}(1, t)=0 .
\end{gathered}
$$

With the help of (1.14) (1.15) (1.16) and (3.1), we deduce

$$
\left|\mathcal{N}_{x}\right|,\left|\mathcal{N}_{x x}\right|,\left|\mathcal{V}_{x}\right|,\left|\mathcal{V}_{x x}\right|,|\psi|,\left|\psi_{x}\right|,|f| \leq O(\delta)
$$

By using (1.6) and (1.11), we arrive at the following equations for $\psi$ and $f$ :

$$
\begin{gathered}
\psi_{t}-\sqrt{f+T_{0}} \psi_{x x}-\frac{\psi_{x}+\mathcal{N}}{2 \sqrt{f+T_{0}}} f_{x}+\frac{\mathcal{V}_{x}}{\sqrt{f+T_{0}}} \psi_{x} \\
-\frac{\sqrt{f+T_{0}} \sqrt{T_{0}} \mathcal{N}_{x}+\mathcal{N} \mathcal{V}_{x}}{\left(f+T_{0}\right) \sqrt{T_{0}}+\sqrt{f+T_{0}} T_{0}} f+\frac{\psi_{x}+\mathcal{N}}{\sqrt{f+T_{0}}} \psi=0 \\
f_{t}-\frac{\sqrt{\left(f+T_{0}\right)^{3}}}{3\left(\psi_{x}+\mathcal{N}\right)}\left(\psi_{x x x}+\mathcal{N}_{x x}\right)-\frac{3}{2} \sqrt{f+T_{0}} f_{x x}-\frac{3 \sqrt{f+T_{0}}}{\psi_{x}+\mathcal{N}} f_{x}\left(\psi_{x x}+\mathcal{N}_{x}\right) \\
-\frac{5}{4 \sqrt{f+T_{0}}} f_{x}^{2}+\frac{\sqrt{f+T_{0}}}{\psi_{x}+\mathcal{N}}\left(\psi_{x x}+\mathcal{N}_{x}\right)\left(\psi+\mathcal{V}_{x}\right)+\frac{3}{2 \sqrt{f+T_{0}}} f_{x}\left(\psi+\mathcal{V}_{x}\right) \\
-\frac{2}{3 \sqrt{f+T_{0}}}\left(\psi+\mathcal{V}_{x}\right)^{2}+\frac{\sqrt{f+T_{0}}}{3}\left(\psi_{x}+\mathcal{V}_{x x}\right)+\frac{f}{\sqrt{f+T_{0}}}=0
\end{gathered}
$$


Multiplying (3.7) by $\psi$, integrating over $[0,1]$ and integrating by parts, we obtain, with the help of (3.3),

$$
\begin{aligned}
\frac{1}{2} \frac{d}{d t} \int_{0}^{1} \psi^{2}+ & \int_{0}^{1} \sqrt{f+T_{0}} \psi_{x}^{2}+\int_{0}^{1} \frac{f_{x}}{2 \sqrt{f+T_{0}}} \psi \psi_{x}-\int_{0}^{1} \frac{\psi_{x}+\mathcal{N}}{2 \sqrt{f+T_{0}}} f_{x} \psi \\
& +\int_{0}^{1} \frac{\mathcal{V}_{x}}{\sqrt{f+T_{0}}} \psi_{x} \psi-\int_{0}^{1} \frac{\sqrt{f+T_{0}} \sqrt{T_{0}} \mathcal{N}_{x}+\mathcal{N} \mathcal{V}_{x}}{\left(f+T_{0}\right) \sqrt{T_{0}}+\sqrt{f+T_{0}} T_{0}} f \psi \\
& +\int_{0}^{1} \frac{\psi_{x}+\mathcal{N}}{\sqrt{f+T_{0}}} \psi^{2}=0
\end{aligned}
$$

By using (3.6), one can show that

$$
\frac{1}{2} \frac{d}{d t} \int_{0}^{1} \psi^{2}+\sqrt{T_{0}} \int_{0}^{1} \psi_{x}^{2}+\frac{\underline{C}}{\sqrt{T_{0}}} \int_{0}^{1} \psi^{2} \leq O(\delta) \int_{0}^{1}\left(\psi_{x}^{2}+\psi^{2}+f^{2}+f_{x}^{2}\right) .
$$

Differentiating (3.7) with respect to $x$, multiplying by $\psi_{x}$, and integrating by parts, we obtain, with the help of (3.4), (3.5) and (1.12),

$$
\begin{aligned}
\frac{1}{2} \frac{d}{d t} & \int_{0}^{1} \psi_{x}^{2}+\int_{0}^{1} \sqrt{f+T_{0}} \psi_{x x}^{2}+\int_{0}^{1} \frac{\psi_{x}+\mathcal{N}}{2 \sqrt{f+T_{0}}} f_{x} \psi_{x x}+\int_{0}^{1} \frac{\mathcal{V}_{x}}{\sqrt{f+T_{0}}} \psi_{x x} \psi_{x} \\
& +\int_{0}^{1} \frac{\mathcal{V}_{x x}}{\sqrt{f+T_{0}}} \psi_{x}^{2}-\int_{0}^{1} \frac{\mathcal{V}_{x}}{2 \sqrt{\left(f+T_{0}\right)^{3}}} f_{x} \psi_{x}^{2}-\int_{0}^{1} \frac{\sqrt{f+T_{0}} \sqrt{T_{0}} \mathcal{N}_{x}+\mathcal{N} \mathcal{V}_{x}}{\left(f+T_{0}\right) \sqrt{T_{0}}+\sqrt{f+T_{0}} T_{0}} f_{x} \psi_{x} \\
& -\int_{0}^{1}\left(\frac{\sqrt{T_{0}} \mathcal{N}_{x} f_{x}}{2\left(\sqrt{\left(f+T_{0}\right)^{3} T_{0}}+\left(f+T_{0}\right) T_{0}\right)}+\frac{\sqrt{\left(f+T_{0}\right.} \mathcal{N}_{x x}+\mathcal{N}_{x} \mathcal{V}_{x}+\mathcal{N} \mathcal{V}_{x x}}{\left(f+T_{0}\right) \sqrt{T_{0}}+\sqrt{f+T_{0}} T_{0}}\right) f \psi_{x} \\
& +\int_{0}^{1}\left(\sqrt{\left(f+T_{0}\right) T_{0}} \mathcal{N}_{x}+\mathcal{N} \mathcal{V}_{x}\right) \frac{2 \sqrt{\left(f+T_{0}\right) T_{0}} f_{x}+T_{0} f_{x}}{2 \sqrt{f+T_{0}}\left(\left(f+T_{0}\right) \sqrt{T_{0}}+\sqrt{f+T_{0}} T_{0}\right)^{2}} f \psi_{x} \\
& +\int_{0}^{1} \frac{\psi_{x}+\mathcal{N}}{\sqrt{f+T_{0}}} \psi_{x}^{2}+\int_{0}^{1} \frac{\psi_{x x}+\mathcal{N}_{x}}{\sqrt{f+T_{0}}} \psi \psi_{x}-\int_{0}^{1} \frac{\psi_{x}+\mathcal{N}}{2 \sqrt{\left(f+T_{0}\right)^{3}}} \psi f_{x} \psi_{x}=0
\end{aligned}
$$

By (3.6) and $\underline{C} \leq \mathcal{N} \leq \bar{C}$, we get

$$
\begin{gathered}
\frac{1}{2} \frac{d}{d t} \int_{0}^{1} \psi_{x}^{2}+\sqrt{T_{0}} \int_{0}^{1} \psi_{x x}^{2}+\int_{0}^{1} \frac{\psi_{x}+\mathcal{N}}{2 \sqrt{f+T_{0}}} f_{x} \psi_{x x}+\frac{\underline{C}}{\sqrt{T_{0}}} \int_{0}^{1} \psi_{x}^{2} \\
\leq O(\delta) \int_{0}^{1}\left(\psi_{x x}^{2}+\psi_{x}^{2}+f^{2}+f_{x}^{2}\right) .
\end{gathered}
$$

Multiplying (3.8) by $f$, integrating over [0,1], and using (3.5), (3.4) and (1.12), we deduce

$$
\begin{aligned}
\frac{1}{2} \frac{d}{d t} \int_{0}^{1} f^{2}+\int_{0}^{1} \frac{\sqrt{\left(f+T_{0}\right)^{3}}}{3\left(\psi_{x}+\mathcal{N}\right)}\left(\psi_{x x}+\mathcal{N}_{x}\right) f_{x}+\int_{0}^{1} \frac{\sqrt{f+T_{0}}\left(\psi_{x x}+\mathcal{N}_{x}\right)}{2\left(\psi_{x}+\mathcal{N}\right)} f_{x} f \\
\quad-\int_{0}^{1} \frac{\sqrt{\left(f+T_{0}\right)^{3}}\left(\psi_{x x}+\mathcal{N}_{x}\right)^{2}}{3\left(\psi_{x}+\mathcal{N}\right)^{2}} f+\int_{0}^{1} \frac{3}{2} \sqrt{f+T_{0}} f_{x}^{2}+\int_{0}^{1} \frac{3}{4 \sqrt{f+T_{0}}} f_{x}^{2} f \\
\quad-\int_{0}^{1} \frac{3 \sqrt{f+T_{0}}}{\psi_{x}+\mathcal{N}} f f_{x}\left(\psi_{x x}+\mathcal{N}_{x}\right)-\int_{0}^{1} \frac{5}{4 \sqrt{f+T_{0}}} f_{x}^{2} f \\
\quad+\int_{0}^{1} \frac{\sqrt{f+T_{0}}}{\psi_{x}+\mathcal{N}}\left(\psi_{x x}+\mathcal{N}_{x}\right)\left(\psi+\mathcal{V}_{x}\right) f+\int_{0}^{1} \frac{3}{2 \sqrt{f+T_{0}}}\left(\psi+\mathcal{V}_{x}\right) f f_{x} \\
\quad-\int_{0}^{1} \frac{2}{3 \sqrt{f+T_{0}}}\left(\psi+\mathcal{V}_{x}\right)^{2} f+\int_{0}^{1} \frac{\sqrt{f+T_{0}}}{3}\left(\psi_{x}+\mathcal{V}_{x x}\right) f+\int_{0}^{1} \frac{f^{2}}{\sqrt{f+T_{0}}}=0
\end{aligned}
$$


By (3.6), the above equation reduces to

$$
\begin{gathered}
\frac{1}{2} \frac{d}{d t} \int_{0}^{1} f^{2}+\int_{0}^{1} \frac{\sqrt{\left(f+T_{0}\right)^{3}}}{3\left(\psi_{x}+\mathcal{N}\right)} \psi_{x x} f_{x}+\int_{0}^{1} \frac{3}{2} \sqrt{f+T_{0}} f_{x}^{2}+\int_{0}^{1} \frac{f^{2}}{\sqrt{f+T_{0}}} \\
\leq O(\delta) \int_{0}^{1}\left(f_{x}^{2}+f^{2}+\psi_{x x}^{2}\right) .
\end{gathered}
$$

It follows from (3.6) and $\underline{C} \leq \mathcal{N} \leq \bar{C}$ that

$$
\frac{1}{2} \frac{d}{d t} \int_{0}^{1} f^{2}+\int_{0}^{1} \frac{\sqrt{\left(f+T_{0}\right)^{3}}}{3\left(\psi_{x}+\mathcal{N}\right)} \psi_{x x} f_{x}+\frac{3}{2} T_{0}^{\frac{1}{2}} \int_{0}^{1} f_{x}^{2}+T_{0}^{-\frac{1}{2}} \int_{0}^{1} f^{2} \leq O(\delta) \int_{0}^{1}\left(\psi_{x x}^{2}+f^{2}+f_{x}^{2}\right)
$$

Since the strong parabolicity condition (1.17) is satisfied, we have

$$
T_{0}^{\frac{1}{2}} \int_{0}^{1}\left[\psi_{x x}^{2}-\left(\frac{T_{0}}{3 \underline{C}}+\frac{\bar{C}}{2 T_{0}}\right)\left|f_{x} \psi_{x x}\right|+f_{x}^{2}\right] \geq T_{0}^{\frac{1}{2}} \lambda \int_{0}^{1}\left(\psi_{x x}^{2}+f_{x}^{2}\right)
$$

Combining (3.10) with (3.11) and using (3.12), we conclude

$$
\begin{gathered}
\frac{d}{d t} \int_{0}^{1}\left(\psi_{x}^{2}+f^{2}\right)+2 T_{0}^{\frac{1}{2}} \lambda \int_{0}^{1}\left(\psi_{x x}^{2}+f_{x}^{2}\right)+\frac{2 \underline{C}}{T_{0}^{\frac{1}{2}}} \int_{0}^{1} \psi_{x}^{2}+\frac{2}{T_{0}^{\frac{1}{2}}} \int_{0}^{1} f^{2} \\
\leq O(\delta) \int_{0}^{1}\left(f_{x}^{2}+f^{2}+\psi_{x}^{2}+\psi_{x x}^{2}\right)
\end{gathered}
$$

Now we can estimate the first derivatives with the help of (3.9) and (3.13),

$$
\frac{d}{d t} \int_{0}^{1}\left(\psi_{x}^{2}+\psi^{2}+f^{2}\right)+a_{1} \int_{0}^{1}\left(\psi_{x x}^{2}+\psi_{x}^{2}+\psi^{2}+f_{x}^{2}+f^{2}\right) \leq 0
$$

where $a_{1}=\min \left\{T_{0}^{\frac{1}{2}} \lambda, T_{0}^{\frac{1}{2}}+\underline{C} T_{0}^{-\frac{1}{2}}, T_{0}^{-\frac{1}{2}}, \underline{C} T_{0}^{-\frac{1}{2}}\right\}$.

Next we proceed to estimate the higher order derivatives. 
Differentiating (3.7) twice with respect to $x$, multiplying by $\psi_{x x}$, using (3.4) and integrating by parts, we obtain

$$
\begin{aligned}
& \frac{1}{2} \frac{d}{d t} \int_{0}^{1} \psi_{x x}^{2}+\int_{0}^{1} \sqrt{f+T_{0}} \psi_{x x x}^{2}+\int_{0}^{1} \frac{f_{x}}{2 \sqrt{f+T_{0}}} \psi_{x x} \psi_{x x x}+\int_{0}^{1} \frac{\psi_{x}+\mathcal{N}}{2 \sqrt{f+T_{0}}} f_{x x} \psi_{x x x} \\
& \quad+\int_{0}^{1} \frac{\psi_{x x}+\mathcal{N}_{x}}{2 \sqrt{f+T_{0}}} f_{x} \psi_{x x x}-\int_{0}^{1} \frac{\psi_{x}+\mathcal{N}}{4 \sqrt{\left(f+T_{0}\right)^{3}}} f_{x}^{2} \psi_{x x x}-\int_{0}^{1} \frac{\mathcal{V}_{x}}{\sqrt{f+T_{0}}} \psi_{x x} \psi_{x x x} \\
& \quad-\int_{0}^{1} \frac{\mathcal{V}_{x x}}{\sqrt{f+T_{0}}} \psi_{x} \psi_{x x x}+\int_{0}^{1} \frac{\mathcal{V}_{x}}{2 \sqrt{\left(f+T_{0}\right)^{3}}} f_{x} \psi_{x} \psi_{x x x} \\
& \quad+\int_{0}^{1} \frac{\sqrt{f+T_{0}} \sqrt{T_{0}} \mathcal{N}_{x}+\mathcal{N} \mathcal{V}_{x}}{\left(f+T_{0}\right) \sqrt{T_{0}}+\sqrt{f+T_{0}} T_{0}} f_{x} \psi_{x x x} \\
&\left.\quad+\int_{0}^{1} \frac{\left(\frac{T_{0}}{2\left(\sqrt{\left(f+T_{0}\right)^{3} T_{0}}+\left(f+T_{x}\right) T_{0}\right)}\right.}{}+\frac{\left.\sqrt{\left(f+T_{0}\right) T_{0}} \mathcal{N}_{x x}+\mathcal{N}_{x} \mathcal{V}_{x}+\mathcal{N} \mathcal{V}_{x x}\right) f \psi_{x x x}}{\left(f+T_{0}\right) \sqrt{T_{0}}+\sqrt{f+T_{0}} T_{0}} f T_{0}\right) T_{0} f_{x}+T_{0} f_{x} \\
&-\int_{0}^{1}\left(\sqrt{\left(f+T_{0}\right) T_{0}} \mathcal{N}_{x}+\mathcal{N} \mathcal{V}_{x}\right) \frac{2 \sqrt{f+T_{0}}\left(\left(f+T_{0}\right) \sqrt{T_{0}}+\sqrt{f+T_{0}} T_{0}\right)^{2}}{2 \sqrt{f}} \psi_{x x x} \\
&-\int_{0}^{1} \frac{\psi_{x}+\mathcal{N}}{\sqrt{f+T_{0}}} \psi_{x} \psi_{x x x}-\int_{0}^{1} \frac{\psi_{x x}+\mathcal{N}_{x}}{\sqrt{f+T_{0}}} \psi \psi_{x x x}+\int_{0}^{1} \frac{\psi_{x}+\mathcal{N}}{2 \sqrt{\left(f+T_{0}\right)^{3}}} \psi f_{x} \psi_{x x x}=0
\end{aligned}
$$

By (3.6),

$$
\begin{aligned}
\frac{1}{2} \frac{d}{d t} \int_{0}^{1} \psi_{x x}^{2} & +\int_{0}^{1} \sqrt{f+T_{0}} \psi_{x x x}^{2}+\int_{0}^{1} \frac{\psi_{x}+\mathcal{N}}{2 \sqrt{f+T_{0}}} f_{x x} \psi_{x x x} \\
& \leq O(\delta) \int_{0}^{1}\left(f_{x}^{2}+\psi_{x x x}^{2}+\psi_{x x}^{2}\right)+\left|I_{1}\right|+\left|I_{2}\right|
\end{aligned}
$$

where

$$
\begin{aligned}
\left|I_{1}\right| & =\left|\int_{0}^{1} \frac{1}{2 \sqrt{f+T_{0}}} f_{x} \psi_{x x} \psi_{x x x}\right| \leq \frac{1}{2 T_{0}^{\frac{1}{2}}}\left|\int_{0}^{1} f_{x}\left(\psi_{x x}^{2}\right)_{x}\right| \\
& \leq \frac{1}{2 T_{0}^{\frac{1}{2}}}\left|\int_{0}^{1} f_{x x} \psi_{x x}^{2}\right| \leq \varepsilon_{1} \int_{0}^{1} f_{x x}^{2}+\frac{1}{8 T_{0}^{\frac{1}{2}} \varepsilon_{1}} \int_{0}^{1} \psi_{x x}^{4} \\
& \leq \varepsilon_{1} \int_{0}^{1} f_{x x}^{2}+\varepsilon_{2} \int_{0}^{1} \psi_{x x x}^{2}+K_{1}\left(\varepsilon_{1}, \varepsilon_{2}\right) \int_{0}^{1} \psi_{x x}^{2}
\end{aligned}
$$

and

$$
\begin{aligned}
\left|I_{2}\right| & =\left|\int_{0}^{1} \frac{\psi_{x}+\mathcal{N}}{4 \sqrt{\left(f+T_{0}\right)^{3}}} f_{x}^{2} \psi_{x x x}\right| \leq \frac{\bar{C}}{2 T_{0}^{\frac{3}{2}}}\left|\int_{0}^{1} f_{x}^{2} \psi_{x x x}\right| \leq \varepsilon_{2} \int_{0}^{1} \psi_{x x x}^{2}+\frac{\bar{C}}{8 T_{0}^{\frac{3}{2}} \varepsilon_{2}} \int_{0}^{1} f_{x}^{4} \\
& \leq \varepsilon_{2} \int_{0}^{1} \psi_{x x x}^{2}+\varepsilon_{1} \int_{0}^{1} f_{x x}^{2}+K_{2}\left(\varepsilon_{1}, \varepsilon_{2}\right) \int_{0}^{1} f_{x}^{2} .
\end{aligned}
$$

In the above calculation, we have used the Gagliardo-Nirenberg inequality to control $I_{1}$ and $I_{2}$. The constants $\varepsilon_{1}$ and $\varepsilon_{2}$ are to be determined. 
Combining (3.15) with (3.16) and (3.17),

$$
\begin{aligned}
& \frac{1}{2} \frac{d}{d t} \int_{0}^{1} \psi_{x x}^{2}+\int_{0}^{1} \sqrt{f+T_{0}} \psi_{x x x}^{2}+\int_{0}^{1} \frac{\psi_{x}+\mathcal{N}}{2 \sqrt{f+T_{0}}} f_{x x} \psi_{x x x} \\
\leq & O(\delta) \int_{0}^{1}\left(f_{x}^{2}+f_{x x}^{2}+\psi_{x x x}^{2}+\psi_{x x}^{2}\right)+2 \varepsilon_{2} \int_{0}^{1} \psi_{x x x}^{2}+2 \varepsilon_{1} \int_{0}^{1} f_{x x}^{2} \\
& +K_{1}\left(\varepsilon_{1}, \varepsilon_{2}\right) \int_{0}^{1} \psi_{x x}^{2}+K_{2}\left(\varepsilon_{1}, \varepsilon_{2}\right) \int_{0}^{1} f_{x}^{2}
\end{aligned}
$$

Differentiating (3.8) with respect to $x$, multiplying by $f_{x}$, using (3.5) and integrating by parts yields

$$
\begin{aligned}
\frac{1}{2} \frac{d}{d t} \int_{0}^{1} & f_{x}^{2}+\int_{0}^{1} \frac{\sqrt{\left(f+T_{0}\right)^{3}}}{3\left(\psi_{x}+\mathcal{N}\right)}\left(\psi_{x x x}+\mathcal{N}_{x x}\right) f_{x x}+\int_{0}^{1} \frac{3}{2} \sqrt{f+T_{0}} f_{x x}^{2} \\
& +\int_{0}^{1} \frac{3 \sqrt{f+T_{0}}}{\psi_{x}+\mathcal{N}} f_{x x} f_{x}\left(\psi_{x x}+\mathcal{N}_{x}\right)+\int_{0}^{1} \frac{5}{4 \sqrt{f+T_{0}}} f_{x}^{2} f_{x x} \\
& -\int_{0}^{1} \frac{\sqrt{f+T_{0}}}{\psi_{x}+\mathcal{N}}\left(\psi_{x x}+\mathcal{N}_{x}\right)\left(\psi+\mathcal{V}_{x}\right) f_{x x}-\int_{0}^{1} \frac{3}{2 \sqrt{f+T_{0}}}\left(\psi+\mathcal{V}_{x}\right) f_{x x} f_{x} \\
& +\int_{0}^{1} \frac{2}{3 \sqrt{f+T_{0}}}\left(\psi+\mathcal{V}_{x}\right)^{2} f_{x x}-\int_{0}^{1} \frac{\sqrt{f+T_{0}}}{3}\left(\psi_{x}+\mathcal{V}_{x x}\right) f_{x x} \\
& -\int_{0}^{1} \frac{1}{\sqrt{f+T_{0}}} f f_{x x}=0
\end{aligned}
$$

Again by (3.6), we get

$$
\begin{aligned}
\frac{1}{2} \frac{d}{d t} \int_{0}^{1} f_{x}^{2}+ & \int_{0}^{1} \frac{\sqrt{\left(f+T_{0}\right)^{3}}}{3\left(\psi_{x}+\mathcal{N}\right)} \psi_{x x x} f_{x x}+\int_{0}^{1} \frac{3}{2} \sqrt{f+T_{0}} f_{x x}^{2} \\
& \leq O(\delta) \int_{0}^{1}\left(\psi_{x x x}^{2}+f_{x x}^{2}+\psi_{x x}^{2}+f_{x}^{2}\right)+\left|I_{3}\right|+\left|I_{4}\right|
\end{aligned}
$$

where

$$
\begin{aligned}
\left|I_{3}\right| & =\left|\int_{0}^{1} \frac{3 \sqrt{f+T_{0}}}{\psi_{x}+\mathcal{N}} f_{x} f_{x x} \psi_{x x}\right| \leq \frac{6 \sqrt{T_{0}}}{\underline{C}}\left|\int_{0}^{1} f_{x} f_{x x} \psi_{x x}\right|=\frac{3 \sqrt{T_{0}}}{\underline{C}}\left|\int_{0}^{1}\left(f_{x}^{2}\right)_{x} \psi_{x x}\right| \\
& \leq \frac{3 \sqrt{T_{0}}}{\underline{C}}\left|\int_{0}^{1} f_{x}^{2} \psi_{x x x}\right| \leq \varepsilon_{2} \int_{0}^{1} \psi_{x x x}^{2}+\frac{3 \sqrt{T_{0}}}{4 \frac{C \varepsilon_{2}}{\int_{0}} \int_{0}^{1} f_{x}^{4}} \\
& \leq \varepsilon_{1} \int_{0}^{1} f_{x x}^{2}+\varepsilon_{2} \int_{0}^{1} \psi_{x x x}^{2}+K_{3}\left(\varepsilon_{1}, \varepsilon_{2}\right) \\
\left|I_{4}\right| & =\left|\int_{0}^{1} \frac{5}{4 \sqrt{f+T_{0}}} f_{x}^{2} f_{x x}\right| \leq \frac{5}{2 T_{0}^{\frac{1}{2}}}\left|\int_{0}^{1} f_{x}^{2} f_{x x}\right| \leq \varepsilon_{1} \int_{0}^{1} f_{x x}^{2}+\frac{5}{8 T_{0}^{\frac{1}{2}} \varepsilon_{1}} \int_{0}^{1} f_{x}^{4} \\
& \leq 2 \varepsilon_{1} \int_{0}^{1} f_{x x}^{2}+K_{4}\left(\varepsilon_{1}\right) \int_{0}^{1} f_{x}^{2}
\end{aligned}
$$


are derived by the techniques used in obtaining (3.16) and (3.17). Thus we obtain

$$
\begin{aligned}
& \frac{1}{2} \frac{d}{d t} \int_{0}^{1} f_{x}^{2}+\int_{0}^{1} \frac{\sqrt{\left(f+T_{0}\right)^{3}}}{3\left(\psi_{x}+\mathcal{N}\right)} \psi_{x x x} f_{x x}+\frac{3}{2} T_{0}^{\frac{1}{2}} \int_{0}^{1} f_{x x}^{2} \\
\leq & O(\delta) \int_{0}^{1}\left(\psi_{x x x}^{2}+f_{x x}^{2}+\psi_{x x}^{2}+f_{x}^{2}\right)+3 \varepsilon_{1} \int_{0}^{1} f_{x x}^{2}+\varepsilon_{2} \int_{0}^{1} \psi_{x x x}^{2} \\
& +K_{3}\left(\varepsilon_{1}, \varepsilon_{2}\right) \int_{0}^{1} f_{x}^{2}+K_{4}\left(\varepsilon_{1}\right) \int_{0}^{1} f_{x}^{2} .
\end{aligned}
$$

Combining (3.18) with (3.21), using the strong parabolicity condition, we obtain

$$
\begin{aligned}
& \frac{d}{d t} \int_{0}^{1}\left(\psi_{x x}^{2}+f_{x}^{2}\right)+T_{0}^{\frac{1}{2}} \lambda \int_{0}^{1}\left(\psi_{x x x}^{2}+f_{x x}^{2}\right) \\
\leq & O(\delta) \int_{0}^{1}\left(\psi_{x x}^{2}+f_{x}^{2}\right)+10 \varepsilon_{1} \int_{0}^{1} f_{x x}^{2}+6 \varepsilon_{2} \int_{0}^{1} \psi_{x x x}^{2}+K \int_{0}^{1}\left(f_{x}^{2}+\psi_{x x}^{2}\right),
\end{aligned}
$$

where $K=\sum_{i=1}^{4} 2 K_{i}$.

$$
\begin{aligned}
& \text { Choosing } \varepsilon_{1}=\frac{T_{0}^{\frac{1}{2}} \lambda}{20} \text { and } \varepsilon_{2}=\frac{T_{0}^{\frac{1}{2}} \lambda}{12} \text {, we have } \\
& \frac{d}{d t} \int_{0}^{1}\left(\psi_{x x}+f_{x}^{2}\right)+\frac{T_{0}^{\frac{1}{2}} \lambda}{2} \int_{0}^{1}\left(\psi_{x x x}^{2}+f_{x x}^{2}\right) \\
& \leq O(\delta) \int_{0}^{1}\left(\psi_{x x}^{2}+f_{x}^{2}\right)+K \int_{0}^{1}\left(f_{x}^{2}+\psi_{x x}^{2}\right) \text {. }
\end{aligned}
$$

Multiplying (3.23) by $\frac{a_{1}}{4 K}$ and adding to (3.14) yields

$\frac{d}{d t} \int_{0}^{1}\left(\left(\psi^{2}+\psi_{x}^{2}+f^{2}\right)+\frac{a_{1}}{4 K}\left(\psi_{x x}^{2}+f_{x}^{2}\right)\right)+a_{2} \int_{0}^{1}\left(\psi^{2}+\psi_{x}^{2}+\psi_{x x}^{2}+f^{2}+f_{x}^{2}+f_{x x}^{2}+\psi_{x x x}^{2}\right) \leq 0$ where $a_{2}=\min \left\{\frac{a_{1}}{2}, \frac{T_{0}^{\frac{1}{2}} \lambda a_{1}}{8 K}\right\}$.

Thus, by Gronwall's inequality,

$$
\|\psi(\cdot, t)\|_{H^{2}}^{2}+\|f(\cdot, t)\|_{H_{1}}^{2} \leq C\left(\|\psi(\cdot, 0)\|_{H^{2}}^{2}+\|f(\cdot, 0)\|_{H^{1}}^{2}\right) \cdot \exp (-\beta t) .
$$

In order to prove Theorem 1.2, we need the following higher order estimate:

LEMma 3.2. There exist positive constants $\delta_{2}>0$ and $\beta_{2}>0$ such that if

$$
\sup _{0 \leq t \leq \tau}\left(\|\psi(\cdot, t)\|_{H^{4}}+\|f(\cdot, t)\|_{H^{3}}\right) \leq \delta_{2}
$$

for any $\tau>0$, then

$$
\|\psi(\cdot, t)\|_{H^{4}}+\|f(\cdot, t)\|_{H^{3}} \leq C\left(\|\psi(\cdot, 0)\|_{H^{4}}+\|f(\cdot, 0)\|_{H^{3}}\right) \exp \left(-\beta_{2} t\right),
$$

for any $t \in[0, \tau]$. 
Proof. By Lemma 3.1, we need to estimate $\left\|\psi_{x x}(\cdot, t)\right\|_{H^{2}}+\left\|f_{x x}(\cdot, t)\right\|_{H^{1}}$. First we estimate $\left\|\psi_{t}(\cdot, t)\right\|_{H^{2}}+\left\|f_{t}(\cdot, t)\right\|_{H^{1}}$, and then we obtain the desired estimate with the help of (3.7) and (3.8).

From (3.3), (3.4), and (3.5), it follows that

$$
\begin{gathered}
\psi_{t}(0, t)=\psi_{t}(1, t)=0, \\
\psi_{x x t}(0, t)=\psi_{x x t}(1, t)=0, \\
f_{x t}(0, t)=f_{x t}(1, t)=0 .
\end{gathered}
$$

By virtue of (1.14) (1.15) (1.16) and (3.25), we obtain

$$
\left|\mathcal{N}_{x}\right|,\left|\mathcal{N}_{x x}\right|,\left|\mathcal{V}_{x}\right|,\left|\mathcal{V}_{x x}\right|,|\psi|,\left|\psi_{x}\right|,\left|\psi_{x x}\right|,\left|\psi_{x x x}\right|,\left|\psi_{t}\right|,\left|\psi_{x t}\right|,|f|,\left|f_{x}\right|,\left|f_{x x}\right|,\left|f_{t}\right| \leq O\left(\delta_{2}\right)
$$

Differentiating (3.7) with respect to $x$,

$$
\psi_{x t}-\sqrt{f+T_{0}} \psi_{x x x}-\frac{\psi_{x}+\mathcal{N}}{2 \sqrt{f+T_{0}}} f_{x x}+\frac{\psi_{x}+\mathcal{N}}{\sqrt{f+T_{0}}} \psi_{x}+j_{1}+j_{2}+j_{3}=0,
$$

where

$$
\begin{aligned}
j_{1}= & -\frac{f_{x}}{2 \sqrt{f+T_{0}}} \psi_{x x}-\frac{\psi_{x x}+\mathcal{N}_{x}}{2 \sqrt{f+T_{0}}} f_{x}+\frac{\psi_{x}+\mathcal{N}}{4 \sqrt{\left(f+T_{0}\right)^{3}}} f_{x}^{2}+\frac{\psi_{x x}+\mathcal{N}_{x}}{\sqrt{f+T_{0}}} \psi \\
& -\frac{\psi_{x}+\mathcal{N}}{2 \sqrt{\left(f+T_{0}\right)^{3}}} \psi f_{x} \\
j_{2}= & \frac{\mathcal{V}_{x}}{\sqrt{f+T_{0}}} \psi_{x x}+\frac{\mathcal{V}_{x x}}{\sqrt{f+T_{0}}} \psi_{x}-\frac{\mathcal{V}_{x}}{2 \sqrt{\left(f+T_{0}\right)^{3}}} \psi_{x} f_{x}, \\
j_{3}= & -\left(\frac{\sqrt{f+T_{0}} \sqrt{T_{0}} \mathcal{N}_{x}+\mathcal{N} \mathcal{V}_{x}}{\left(f+T_{0}\right) \sqrt{T_{0}}+\sqrt{f+T_{0}} T_{0}}\right)_{x} f-\frac{\sqrt{f+T_{0}} \sqrt{T_{0}} \mathcal{N}_{x}+\mathcal{N} \mathcal{V}_{x}}{\left(f+T_{0}\right) \sqrt{T_{0}}+\sqrt{f+T_{0}} T_{0}} f_{x} .
\end{aligned}
$$

Differentiating (3.31) with respect to $t$,

$$
\psi_{x t t}-\sqrt{f+T_{0}} \psi_{x x x t}-\frac{\psi_{x}+\mathcal{N}}{2 \sqrt{f+T_{0}}} f_{x x t}+\frac{\psi_{x}+\mathcal{N}}{\sqrt{f+T_{0}}} \psi_{x t}+j_{4}+\left(j_{1}\right)_{t}+\left(j_{2}\right)_{t}+\left(j_{3}\right)_{t}=0,
$$

where

$$
j_{4}=-\frac{f_{t}}{2 \sqrt{f+T_{0}}} \psi_{x x x}-\frac{\psi_{x t} f_{x x}}{2 \sqrt{f+T_{0}}}+\frac{\psi_{x}+\mathcal{N}}{4 \sqrt{\left(f+T_{0}\right)^{3}}} f_{t} f_{x x}+\frac{\psi_{x t} \psi_{x}}{\sqrt{f+T_{0}}}-\frac{\psi_{x}+\mathcal{N}}{2 \sqrt{\left(f+T_{0}\right)^{3}}} f_{t} \psi_{x} .
$$

Multiplying $(3.33)$ by $\psi_{x t}$, integrating over $[0,1]$, integrating by parts, and using $(3.28)$ and (3.29), we obtain

$$
\frac{1}{2} \frac{d}{d t} \int_{0}^{1} \psi_{x t}^{2}+\int_{0}^{1}\left(\sqrt{f+T_{0}} \psi_{x x t}^{2}+\frac{\psi_{x}+\mathcal{N}}{2 \sqrt{f+T_{0}}} f_{x t} \psi_{x x t}\right)+\int_{0}^{1} \frac{\psi_{x}+\mathcal{N}}{\sqrt{f+T_{0}}} \psi_{x t}^{2}=\sum_{i=1}^{5} J_{i}
$$


where, by virtue of $(3.30)$,

$$
\begin{aligned}
J_{1} & =-\int_{0}^{1}\left(j_{1}\right)_{t} \psi_{x t}=O\left(\delta_{2}\right) \int_{0}^{1} \psi_{x x t}^{2}+f_{x t}^{2}+\psi_{x t}^{2}, \\
J_{2} & =-\int_{0}^{1}\left(j_{2}\right)_{t} \psi_{x t}=O\left(\delta_{2}\right) \int_{0}^{1} \psi_{x x t}^{2}+f_{x t}^{2}+\psi_{x t}^{2}, \\
J_{3} & =-\int_{0}^{1}\left(j_{3}\right)_{t} \psi_{x t}=O\left(\delta_{2}\right) \int_{0}^{1} f_{x t}^{2}+\psi_{x t}^{2}, \\
J_{4} & =-\int_{0}^{1} j_{4} \psi_{x t}=O\left(\delta_{2}\right) \int_{0}^{1} \psi_{x t}^{2}, \\
J_{5} & =-\int_{0}^{1} \frac{f_{x}}{2 \sqrt{f+T_{0}}} \psi_{x t} \psi_{x x t}-\int_{0}^{1} \frac{\psi_{x x}+\mathcal{N}_{x}}{2 \sqrt{f+T_{0}}} f_{x t} \psi_{x t}+\int_{0}^{1} \frac{\psi_{x}+\mathcal{N}}{4 \sqrt{\left(f+T_{0}\right)^{3}}} f_{x} f_{x t} \psi_{x t} \\
& =O\left(\delta_{2}\right) \int_{0}^{1} \psi_{x x t}^{2}+f_{x t}^{2}+\psi_{x t}^{2} .
\end{aligned}
$$

Hence

$$
\begin{gathered}
\frac{1}{2} \frac{d}{d t} \int_{0}^{1} \psi_{x t}^{2}+\int_{0}^{1}\left(\sqrt{f+T_{0}} \psi_{x x t}^{2}+\frac{\psi_{x}+\mathcal{N}}{2 \sqrt{f+T_{0}}} f_{x t} \psi_{x x t}\right)+\int_{0}^{1} \frac{\psi_{x}+\mathcal{N}}{\sqrt{f+T_{0}}} \psi_{x t}^{2} \\
=O\left(\delta_{2}\right) \int_{0}^{1} \psi_{x x t}^{2}+f_{x t}^{2}+\psi_{x t}^{2} .
\end{gathered}
$$

Differentiating (3.8) with respect $t$,

$$
f_{t t}-\frac{3}{2} \sqrt{f+T_{0}} f_{x x t}-\frac{\sqrt{\left(f+T_{0}\right)^{3}}}{3\left(\psi_{x}+\mathcal{N}\right)} \psi_{x x x t}+\frac{1}{\sqrt{f+T_{0}}} f_{t}+\sum_{i=6}^{12} j_{i}=0
$$

where

$$
\begin{aligned}
j_{6}=- & \frac{\sqrt{f+T_{0}}}{2\left(\psi_{x}+\mathcal{N}\right)}\left(\psi_{x x x}+\mathcal{N}_{x x}\right) f_{t}+\frac{\sqrt{\left(f+T_{0}\right)^{3}}}{3\left(\psi_{x}+\mathcal{N}\right)^{2}}\left(\psi_{x x x}+\mathcal{N}_{x x}\right) \psi_{x t} \\
& -\frac{3}{4 \sqrt{f+T_{0}}} f_{x x} f_{t}-\frac{f f_{t}}{2 \sqrt{\left(f+T_{0}\right)^{3}}}, \\
j_{7}=- & \frac{3 \sqrt{f+T_{0}}}{\psi_{x}+\mathcal{N}} f_{x} \psi_{x x t}-\frac{3 \sqrt{f+T_{0}}}{\psi_{x}+\mathcal{N}} f_{x t}\left(\psi_{x x}+\mathcal{N}_{x}\right) \\
& -\frac{3\left(\psi_{x x}+\mathcal{N}_{x}\right)}{2\left(\psi_{x}+\mathcal{N}\right) \sqrt{f+T_{0}}} f_{t} f_{x}+\frac{3 \sqrt{f+T_{0}}}{\left(\psi_{x}+\mathcal{N}\right)^{2}} f_{x} \psi_{x t}\left(\psi_{x x}+\mathcal{N}_{x}\right), \\
j_{8}=- & \frac{10 f_{x} f_{x t}}{4 \sqrt{f+T_{0}}}-\frac{5 f_{x}^{2} f_{t}}{8 \sqrt{\left(f+T_{0}\right)^{3}}}, \\
j_{9}= & \frac{\sqrt{f+T_{0}}}{\psi_{x}+\mathcal{N}}\left(\psi_{x x}+\mathcal{N}_{x}\right) \psi_{t}+\frac{\sqrt{f+T_{0}}}{\psi_{x}+\mathcal{N}} \psi_{x x t}\left(\psi+\mathcal{V}_{x}\right) \\
& +\frac{\left(\psi_{x x}+\mathcal{N}_{x}\right)\left(\psi+\mathcal{V}_{x}\right)}{2 \sqrt{f+T_{0}}\left(\psi_{x}+\mathcal{N}\right)} f_{t}-\frac{\sqrt{f+T_{0}}}{\left(\psi_{x}+\mathcal{N}\right)^{2}} \psi_{x t}\left(\psi_{x x}+\mathcal{N}_{x}\right)\left(\psi+\mathcal{V}_{x}\right), \\
j_{10}= & \frac{3 f_{x} \psi_{t}}{2 \sqrt{f+T_{0}}}+\frac{3 f_{x t}\left(\psi+\mathcal{V}_{x}\right)}{2 \sqrt{f+T_{0}}}-\frac{3 f_{x}\left(\psi+\mathcal{V}_{x}\right)}{4 \sqrt{\left(f+T_{0}\right)^{3}}} f_{t} \\
j_{11}= & -\frac{4\left(\psi+\mathcal{V}_{x}\right)}{3 \sqrt{f+T_{0}}}+\frac{\left(\psi+\mathcal{V}_{x}\right)^{2}}{3 \sqrt{\left(f+T_{0}\right)^{3}}} f_{t} \\
j_{12}= & \frac{\sqrt{f+T_{0}}}{3} \psi_{x t}+\frac{\psi_{x}+\mathcal{V}_{x x}}{6 \sqrt{f+T_{0}}} f_{t} .
\end{aligned}
$$


Multiplying (3.38) by $f_{t}$, integrating over $[0,1]$, and integrating by parts,

$$
\frac{1}{2} \frac{d}{d t} \int_{0}^{1} f_{t}^{2}+\int_{0}^{1}\left(\frac{3}{2} \sqrt{f+T_{0}} f_{x t}^{2}+\frac{\sqrt{\left(f+T_{0}\right)^{3}}}{3\left(\psi_{x}+\mathcal{N}\right)} \psi_{x x t} f_{x t}\right)+\int_{0}^{1} \frac{1}{\sqrt{f+T_{0}}} f_{t}^{2}=\sum_{i=6}^{13} J_{i}
$$

where, by account of $(3.30)$, we have

$$
\begin{aligned}
& J_{6}+J_{11}+J_{12}=-\int_{0}^{1}\left(j_{6}+j_{11}+j_{12}\right) f_{t}=O\left(\delta_{2}\right) \int_{0}^{1} f_{t}^{2}, \\
& J_{7}=-\int_{0}^{1} j_{7} f_{t}=O\left(\delta_{2}\right) \int_{0}^{1}\left(f_{x t}^{2}+\psi_{x x t}^{2}+f_{t}^{2}\right), \\
& J_{8}+J_{10}=-\int_{0}^{1}\left(j_{8}+j_{10}\right) f_{t}=O\left(\delta_{2}\right) \int_{0}^{1}\left(f_{x t}^{2}+f_{t}^{2}\right), \\
& J_{9}=-\int_{0}^{1} j_{9} f_{t}=O\left(\delta_{2}\right) \int_{0}^{1}\left(\psi_{x x t}^{2}+f_{t}^{2}\right), \\
& J_{13}=-\int_{0}^{1} \frac{3}{4 \sqrt{f+T_{0}}} f_{x} f_{x t} f_{t}-\int_{0}^{1} \frac{\sqrt{f+T_{0}}}{2\left(\psi_{x}+\mathcal{N}\right)} f_{x} \psi_{x x t} f_{t} \\
& \quad+\int_{0}^{1} \frac{\sqrt{\left(f+T_{0}\right)^{3}}}{3\left(\psi_{x}+\mathcal{N}\right)^{2}}\left(\psi_{x x}+\mathcal{N}_{x}\right) \psi_{x x t} f_{t} \\
& =O\left(\delta_{2}\right) \int_{0}^{1}\left(f_{x t}^{2}+\psi_{x x t}^{2}+f_{t}^{2}\right) .
\end{aligned}
$$

We have thus obtained

$$
\begin{gathered}
\frac{1}{2} \frac{d}{d t} \int_{0}^{1} f_{t}^{2}+\int_{0}^{1}\left(\frac{3}{2} \sqrt{f+T_{0}} f_{x t}^{2}+\frac{\sqrt{\left(f+T_{0}\right)^{3}}}{3\left(\psi_{x}+\mathcal{N}\right)} \psi_{x x t} f_{x t}\right)+\int_{0}^{1} \frac{1}{\sqrt{f+T_{0}}} f_{t}^{2} \\
=O\left(\delta_{2}\right) \int_{0}^{1}\left(f_{x t}^{2}+\psi_{x x t}^{2}+f_{t}^{2}\right) .
\end{gathered}
$$

Combining (3.37) with (3.42), and with the help of (1.17), we deduce

$$
\begin{aligned}
\frac{d}{d t} \int_{0}^{1}\left(\psi_{x t}^{2}+f_{t}^{2}\right)+ & 2 T_{0}^{\frac{1}{2}} \lambda \int_{0}^{1}\left(\psi_{x x t}^{2}+f_{x t}^{2}\right)+\frac{2 \underline{C}}{T_{0}^{\frac{1}{2}}} \int_{0}^{1} \psi_{x t}^{2}+\frac{2}{T_{0}^{\frac{1}{2}}} \int_{0}^{1} f_{t}^{2} \\
& =O\left(\delta_{2}\right) \int_{0}^{1}\left(f_{x t}^{2}+\psi_{x x t}^{2}+f_{t}^{2}\right) .
\end{aligned}
$$

We have thus completed the first step by establishing the estimate

$$
\frac{d}{d t} \int_{0}^{1}\left(\psi_{x t}^{2}+f_{t}^{2}\right)+a_{3} \int_{0}^{1}\left(\psi_{x x t}^{2}+f_{x t}^{2}+\psi_{x t}^{2}+f_{t}^{2}\right) \leq 0,
$$

where $a_{3}=\min \left\{T_{0}^{\frac{1}{2}}, \underline{C} / T_{0}^{\frac{1}{2}}, 1 / T_{0}^{\frac{1}{2}}\right\}$.

Differentiating (3.33) with respect to $x$, multiplying by $\psi_{x x t}$, integrating over $[0,1]$, and integrating by parts, we obtain, with the help of (3.28),

$\frac{1}{2} \frac{d}{d t} \int_{0}^{1} \psi_{x x t}^{2}+\int_{0}^{1}\left(\sqrt{f+T_{0}} \psi_{x x x t}^{2}+\frac{\psi_{x}+\mathcal{N}}{2 \sqrt{f+T_{0}}} f_{x x t} \psi_{x x x t}\right)+\int_{0}^{1} \frac{\psi_{x}+\mathcal{N}}{\sqrt{f+T_{0}}} \psi_{x x t}^{2}=\sum_{i=1}^{5} H_{i}$, 
where, by virtue of $(3.30)$,

$$
\begin{aligned}
& H_{1}=\int_{0}^{1}\left(j_{1}\right)_{t} \psi_{x x x t}=O\left(\delta_{2}\right) \int_{0}^{1} \psi_{x x x t}^{2}+\psi_{x x t}^{2}+f_{x t}^{2} \\
& H_{2}=\int_{0}^{1}\left(j_{2}\right)_{t} \psi_{x x x t}=O\left(\delta_{2}\right) \int_{0}^{1} \psi_{x x x t}^{2}+\psi_{x x t}^{2}+f_{x t}^{2} \\
& H_{3}=\int_{0}^{1}\left(j_{3}\right)_{t} \psi_{x x x t}=O\left(\delta_{2}\right) \int_{0}^{1} \psi_{x x x t}^{2}+f_{x t}^{2} \\
& H_{4}=\int_{0}^{1} j_{4} \psi_{x x x t}=O\left(\delta_{2}\right) \int_{0}^{1} \psi_{x x x t}^{2} \\
& H_{5}=-\int_{0}^{1} \frac{\psi_{x x}+\mathcal{N}_{x}}{\sqrt{f+T_{0}}} \psi_{x t} \psi_{x x t}+\int_{0}^{1} \frac{\psi_{x}+\mathcal{N}}{2 \sqrt{\left(f+T_{0}\right)^{3}}} f_{x} \psi_{x t} \psi_{x x t}=O\left(\delta_{2}\right) \int_{0}^{1} \psi_{x x t}^{2}
\end{aligned}
$$

In the above process, it is not necessary to use high order derivatives such as $\psi_{x x x t}, f_{x x t}$, as we can use difference quotients instead.

Differentiating (3.38) with respect to $x$, multiplying by $f_{x t}$, integrating over $[0,1]$, integrating by parts, and recalling (3.29), we get

$$
\frac{1}{2} \frac{d}{d t} \int_{0}^{1} f_{x t}^{2}+\int_{0}^{1}\left(\frac{3}{2} \sqrt{f+T_{0}} f_{x x t}^{2}+\frac{\sqrt{\left(f+T_{0}\right)^{3}}}{3\left(\psi_{x}+\mathcal{N}\right)} \psi_{x x x t} f_{x x t}\right)+\int_{0}^{1} \frac{1}{\sqrt{f+T_{0}}} f_{x t}^{2}=\sum_{i=6}^{13} H_{i}
$$

where we have, by virtue of (3.30),

$$
\begin{aligned}
& H_{6}+H_{8}+H_{10}+H_{11}+H_{12}=\int_{0}^{1}\left(j_{6}+j_{8}+j_{10}+j_{11}+j_{12}\right) f_{x x t}=O\left(\delta_{2}\right) \int_{0}^{1} f_{x x t}^{2}, \\
& H_{7}+H_{9}=\int_{0}^{1}\left(j_{7}+j_{9}\right) f_{x x t}=O\left(\delta_{2}\right) \int_{0}^{1}\left(f_{x x t}^{2}+\psi_{x x t}^{2}\right), \\
& H_{13}=\int_{0}^{1} \frac{1}{2 \sqrt{\left(f+T_{0}\right)^{3}}} f_{x} f_{t} f_{x t}=O\left(\delta_{2}\right) \int_{0}^{1} f_{x t}^{2} .
\end{aligned}
$$

Combining (3.45) with (3.47) and (1.17), we obtain our second estimate

$$
\frac{d}{d t} \int_{0}^{1}\left(\psi_{x x t}^{2}+f_{x t}^{2}\right)+a_{3} \int_{0}^{1}\left(\psi_{x x x t}^{2}+f_{x x t}^{2}+\psi_{x x t}^{2}+f_{x t}^{2}\right) \leq 0
$$

We can calculate $\psi_{x x x x}, f_{x x x}$ directly by (3.7) and (3.8), in terms of $\psi_{x x t}, f_{x t}$ and their lower order derivatives. Thus, combining the above results with Lemma 3.1 we complete the proof of Theorem 1.2.

Acknowledgement. We thank Prof. Dafermos for his great help and useful advice.

\section{REFERENCES}

[1] N. Ben Abdallah and P. Degond, On a hierarchy of macroscopic models for semiconductor, J. Math. Phys., 37 (1996), 3383-3333.

[2] N. Ben Abdallah, P. Degond, and S. Génieys, An energy-transport model for semiconductors derived from the Boltzmann equation, J. Stat. Phys., 84 (1996), 205-231.

[3] E. Lyumkis, B. Polsky, A. Shur, and D. Visocky, Transient semiconductor device simulation including energy balance equation, Compel, 11 (1992), 311-325.

[4] L. Chen and L. Hsiao, Mixed Boundary Value Problem of Stationary Energy Transport Model, preprint. 
[5] L. Chen and L. Hsiao, The Solution of Lyumkis Energy Transport Model in Semiconductor Science, preprint.

[6] P. Degond, S. Génieys, and A. Jüngel, A steady-state system in nonequilibrium thermodynamics including thermal and electrical effects, Math. Meth. Appl. Sci., 21 (1998) 1399-1413.

[7] P. Degond, S. Génieys, and A. Jüngel, A system of parabolic equations in nonequilibrium thermodynamics including thermal and electrical effects, J. Math. Pures Appl. 76 (1997), 991-1015.

[8] S. Génieys, Energy transport model for a non degenerate semiconductor. Convergence of the Hilbert expansion in the linearized case, Asympt. Anal., 17 (1998), 279-308.

[9] J. A. Griepentrog, An application of the implicit function theorem to an energy model of the semiconductor theory, Z. Angew. Math. Mech., 79 (1999), 43-51.

[10] L. Hsiao and T. Yang, Asymptotics of initial boundary value problems for hydrodynamic and drift diffusion models for semiconductors, J. Diff. Eqns., 170 (2001), 472-493.

[11] A. Jüngel, Regularity and uniqueness of solutions to a parabolic system in nonequilibrium thermodynamics, Nonlin. Anal. 41 (2000), 669-688.

[12] A. Jüngel, Quasi-hydrodynamic semiconductor equations, Basel; Boston; Berlin; Birkhäuser, 2001.

[13] A. Jüngel, Macroscopic models for semiconductor devices. A review, preprint.

[14] H. Xie, $\mathcal{L}^{2, \mu}(\Omega)$ estimate to the mixed boundary value problem for second order elliptic equations and its application in the thermistor problem, Nonlinear Anal. 24, (1995), 9-27. 